Article

\title{
Investigating the Relationship of Genotype and Geographical Location on Volatile Composition and Sensory Profile of Celery (Apium graveolens)
}

\author{
Lucy Turner $^{1}$ D, Stella Lignou ${ }^{1, * \mathbb{D}}$, Frances Gawthrop ${ }^{2}$ and Carol Wagstaff ${ }^{1}$ (D) \\ 1 Department of Food and Nutritional Sciences, University of Reading, Harry Nursten Building, Whiteknights, \\ Reading RG6 6DZ, UK; L.Turner@pgr.reading.ac.uk (L.T.); c.wagstaff@reading.ac.uk (C.W.) \\ 2 A.L. Tozer Ltd., Pyports, Downside Bridge Road, Cobham KT11 3EH, UK; frances.gawthrop@tozerseeds.com \\ * Correspondence: s.lignou@reading.ac.uk; Tel.: +44-(0)118-378-8717
}

\section{check for}

updates

Citation: Turner, L.; Lignou, S.; Gawthrop, F.; Wagstaff, C. Investigating the Relationship of Genotype and Geographical Location on Volatile Composition and Sensory Profile of Celery (Apium graveolens). Int. J. Mol. Sci. 2021, 22, 12016. https://doi.org/10.3390/ ijms222112016

Academic Editors: Salvador Rosello and Jaime Cebolla-Cornejo

Received: 4 October 2021

Accepted: 2 November 2021

Published: 6 November 2021

Publisher's Note: MDPI stays neutral with regard to jurisdictional claims in published maps and institutional affiliations.

Copyright: (c) 2021 by the authors. Licensee MDPI, Basel, Switzerland. This article is an open access article distributed under the terms and conditions of the Creative Commons Attribution (CC BY) license (https:// creativecommons.org/licenses/by/ 4.0/)
Abstract: Numerous varieties of celery are grown in multiple countries to maintain supply, demand and availability for all seasons; thus, there is an expectation for a consistent product in terms of taste, flavour, and overall quality. Differences in climate, agronomy and soil composition will all contribute to inconsistencies. This study investigated the volatile and sensory profile of eight celery genotypes grown in the UK (2018) and Spain (2019). Headspace analysis determined the volatile composition of eight genotypes, followed by assessment of the sensory profile using a trained panel. Significant differences in the volatile composition and sensory profile were observed; genotype and geographical location both exerted influences. Two genotypes exhibited similar aroma composition and sensory profile in both locations, making them good candidates to drive breeding programmes aimed at producing varieties that consistently display these distinctive sensory properties. Celery samples harvested in the UK exhibited a higher proportion of sesquiterpenes and phthalides, whereas samples harvested in Spain expressed a higher aldehyde and ketone content. Studying the relationship between growing environment and genotype will provide information to guide growers in how to consistently produce a high-quality crop.

Keywords: celery; aroma; volatile compounds; SPME GCMS; phthalides; terpenes; harvest

\section{Introduction}

Apium graveolens, commonly known as celery, is a vegetable with long fibrous stalks, belonging to the Apiaceae or Umbelliferae family, characterised by its discoid or 'umbrella' shaped flowers, known as umbels. Similar to other members of the Apiaceae family, including carrots, coriander and parsley, celery possesses a strong, distinct flavour profile, placing it as a key component in soups, stocks and sauces [1,2]. Compounds that constitute the aroma profile include a range of monoterpenes (myrcene, limonene, $\beta$-pinene and $\gamma$-terpinene), sesquiterpenes ( $\beta$-caryophyllene, $\alpha$-humulene, $\alpha$ - and $\beta$-selinene) and phthalides (sedanenolide, neocnidilide and 3-n-butylphthalide) [2-7]. The latter compounds have been reported throughout the literature to be the characteristic odour compounds of celery [7], with odour characteristics identified by Turner, Dawda, Gawthrop, Wagstaff and Lignou [8] of 'celery', 'cooked celery' and 'herbal'. Celery has long been grown and consumed globally and, for this reason, the aroma profile has been studied using a range of cultivars, grown in a variety of years and geographical locations, and analysed using extraction methods including solvent assisted flavour extraction (SAFE) and solid phase microextraction (SPME) which are, most typically, followed by gas chromatography/mass spectrometry (GCMS) [3-6,8]. Possibly the earliest investigation, completed by Gold and Wilson [9], determined the volatile composition of celery juice using distillation followed by gas chromatography. This identified a collection of compounds ranging from aldehydes, esters, alcohols and, most importantly, phthalides. More recently completed work not only 
confirms the compounds identified by Gold and Wilson [9] but displays the complex aroma profile of celery and the variety of compound groups that comprise the aroma profile [7].

As a commonly used vegetable, there is an expectation for celery to be available continuously for consumers; however, in countries such as the United Kingdom, this is not possible due to the unfavourable winter conditions. During the summer months, celery can be grown in the UK as the environment is suitable for growth and, often, celery can continue to be grown on the east coast through autumn. Nevertheless, the annual consumer demand for celery is not met. To combat this issue, celery is grown in warmer locations, such as southern Spain, where they are packaged and processed and then transported to UK retailers. Although offering a solution to meet the demand, utilising seasons in Spain means growing in arid and semi-arid conditions, requiring different agronomy compared to that needed for the UK's growing environment, and thus creating inconsistencies within the aroma quality of the celery produce available. While not thoroughly understood within celery, the influence of abiotic and biotic factors upon the aroma of crops in general has been investigated by others, and differences have been observed [7,10-13]. Exposure to different stresses such as temperature, relative humidity, soil and water compositions have been shown to influence the production of primary and secondary metabolites, ultimately leading to variation within the volatile composition $[7,10]$. Previously, Turner, Lignou, Gawthrop and Wagstaff [10] observed significant differences in the volatile composition and sensory profile of eight celery genotypes grown in the same geographical location in 2018 and 2020. Despite the genotypes displaying significant interactions, it was the differences in environment over the two seasons that had a stronger influence over the volatile composition of celery. The review recently completed by the authors [7] combined data from previously published experiments that investigated the aroma profile of celery, identifying missing data through the exclusion of information, including cultivar name, origin, location of growth, harvest year and conditions of growth Exposing variation in the presence or absence of compounds and their composition within celery, the authors concluded that without stating all experimental information, the data became unrepeatable. To overcome this, the authors put forward the Minimum Information About a Plant Aroma Experiment (MIAPAE), inviting authors to include parameters used during preharvest, harvest and postharvest as well as extraction and analysis methods, allowing for the building of a repository whereby aroma data for plants can repeated and interpreted correctly [7].

Albeit limited, investigations exploring the impact of geographical locations on celery have been completed; Marongiu et al. [11] compared the volatile composition of wild celery grown and collected in Portugal and Italy as well as using different extraction methods (super critical fluid extraction and hydrodistillation). Differences in the composition caused by both the geographical location and extraction method were observed. Phthalide compounds including sedanenolide and neocnidilide expressed significant differences according to these factors, ultimately concluding that environmental differences between Portugal and Italy were the main cause of observed compositional differences. The cultivar of the wildtype celery used in this study was not included, nor were differences in agricultural techniques and growing environments. However, observed variances in the aroma composition in celery caused by these factors have previously been displayed. Rożek, Nurzyńska-Wierda and Kosior [12] identified that drought stress led to an increase in essential oil due to an increase in the production of secondary metabolites, whereas van Wassenhove, Dirinck, Schamp and Vulsteke [13] observed changes in the phthalide and terpene content when nitrogenous fertiliser (organic and/or inorganic) was applied to celery.

This study aims to investigate the relationship between genotype and geographical location of cultivation upon the volatile composition of eight celery varieties grown in Ely, UK in 2018 and Aguilas, Spain in 2019. Sensory evaluation using a trained panel was completed to understand how chemical and physiological changes lead to differences in the organoleptic perception and to identify interactions between compound groups 
and geographical location. Ultimately, this information can be used to assist breeders and growers to develop and select cultivars that are optimal for specific growing environments, to produce a consistently flavoured product. Although factors such as temperature and relative humidity are uncontrollable, growers can apply organic/inorganic fertilisers, herbicides/fungicides and supplementary irrigation to aid optimal conditions for celery growth.

\section{Materials and Methods}

\subsection{Celery Material and MIAPAE Standard}

2.1.1. Sample Information

The eight parental celery genotypes used in these field trials were chosen due to their differences in physical and chemical attributes. Although commercial confidentiality precludes revealing the exact genetic identity of each line used in this paper, the origins of these parental breeding lines and their image postharvest can be found in Supplementary Material (Table S1). Prior to GC/MS analysis, celery material was freeze-dried to ensure consistent aroma quality throughout instrumental analysis. As expected, volatile loss was observed between fresh and freeze-dried samples, however, consistency in relative amount was observed throughout repetitions and the most reported compounds were also identified. Freeze-drying is a method that has been used previously to preserve the volatile content of herbs [14-16], and, furthermore, Hoffman [17] identified freeze-drying as a preservation method that best retains a typical aroma at a strong intensity.

\subsubsection{Timing, Location and Environment}

Celery seed (Apium graveolens) of eight parental genotypes supplied by Tozer Seeds Ltd. (Cobham, United Kingdom) were grown in commercial conditions and harvested in Cambridgeshire (United Kingdom) by G's Fresh Ltd. (Ely, United Kingdom $\left(52^{\circ} 21^{\prime} 12.9^{\prime \prime} \mathrm{N}\right.$ $\left.0^{\circ} 17^{\prime} 15.6^{\prime \prime} \mathrm{E}\right)$ ) during spring/summer 2018. In 2019, the same eight parental varieties of celery were grown and harvested in Aguilas, Spain by G's España Ltd. $\left(37^{\circ} 25^{\prime} 43.2^{\prime \prime} \mathrm{N}\right.$ $\left.1^{\circ} 39^{\prime} 56.2^{\prime \prime} \mathrm{W}\right)$.

Celery grown in the UK was grown on sandy loam soils with naturally high groundwater and a peaty surface, whereas celery grown in Spain was grown on Calcisol soils. Both harvests were grown in a randomised block design, using commercial celery products as border plants to remove edge effects and subjected to the same commercial conditions including application of agronomic techniques, fertilizer and irrigation as commercial celery. For both years, $20-25 \mathrm{~mm}$ of overhead irrigation was used every four days, and standard commercial fertiliser, pest and disease control regimes were applied. In 2018, plugs were transplanted mid-June after 22 days' growth in the nursery, then harvested 91 days later. The average daily air temperature was $18.2^{\circ} \mathrm{C}$, with $0.2 \mathrm{~mm}$ of rainfall daily and an average relative humidity of $88.1 \%$. Average wind speed was $1.9 \mathrm{~ms}$ and the dew point was $15.5^{\circ} \mathrm{C}$. In 2019 , plugs were transplanted in early January after growing for 20 days in the nursery, then harvested in late March, 87 days later. The average daily air temperature was $17.6^{\circ} \mathrm{C}$, with $0.4 \mathrm{~mm}$ of average rainfall and an average relative humidity of $77.3 \%$. Average wind speed was $1.7 \mathrm{~ms}$ and dew point was $6.0^{\circ} \mathrm{C}$. Prior to harvest, the celery was subject to regular in-field assessment to ensure standards for commercial quality were met, including visual and taste tests. These celeries were harvested within a close timeframe of the commercial produce also being grown in the field, which acted as an indicator for the appropriate commercial harvest maturity.

\subsubsection{Raw Material Collection, Processing Storage}

The celery was grown at a density of 10 plants $\mathrm{m}^{-2}$, and three replicates were harvested from each block using a celery knife. Celery petioles were cut to $20 \mathrm{~cm}$, discarding outer petioles, the base, leaves and any knuckles, and sealed in labelled bags for transportation to the University of Reading (United Kingdom). Harvesting in Spain followed the same procedure; however, celery was packed into cool boxes and transported to the UK 
in refrigerated conditions using G's Fresh Ltd. courier. Transportation took two days and samples were collected from G's Fresh (Ely, Cambridgeshire, UK) before transportation back to the University of Reading.

Celery samples used for sensory evaluation were refrigerated for one day before presenting to the trained panel, whereas samples for aroma analysis were immediately frozen at $-80^{\circ} \mathrm{C}$ for one week and subsequently freeze-dried for five days. Samples were then milled to a fine powder using a milling machine (Thomas Scientific, Swedesboro, NJ, USA) and stored in an airtight container for a maximum of two weeks before analysis with gas chromatography/mass spectrometry (GC/MS).

\subsection{Chemicals Reagents}

For GC/MS analysis, calcium chloride and the alkane standard $C_{6}-C_{25}\left(100 \mu \mathrm{g} \mathrm{mL}{ }^{-1}\right)$ in diethyl ether were obtained from Merck (Poole, UK).

\subsection{Volatile Analysis Using SPME GCMS}

For headspace sampling, the celery sample $(0.5 \mathrm{~g})$ was combined with $0.5 \mathrm{~mL}$ of saturated calcium chloride solution and filled to $5 \mathrm{~mL}$ using HPLC-grade water in a $15 \mathrm{~mL}$ SPME vial fitted with a screw cap. Samples were analysed by automated headspace SPME using an Agilent 110 PAL injection system and Agilent 7890 gas chromatograph with 5975C mass spectrometer (Agilent, Santa Clara, CA, USA) according to Turner et al. [8,10].

\subsection{Sensory Evaluation of Fresh Celery Samples}

Sensory evaluation was carried out using quantitative descriptive analysis (QDA ${ }^{\mathrm{TM}}$ ) to determine the sensory characteristics of the eight celery samples, and the characteristics were estimated quantitatively. The trained sensory panel at the Sensory Science Centre (University of Reading, $\mathrm{n}=12 ; 11$ female and 1 male) was used to develop a consensus vocabulary to describe the sensory characteristics of the eight celery genotypes. The terms were discussed by the panellists as a group, facilitated by a panel leader, and this led to a consensus of 22 and 23 attributes for the UK and Spanish harvest, respectively. The sensory assessment of the samples was carried out according to Turner et al. [8] at the Sensory Science Centre (University of Reading) using Compusense Cloud Software (Version 21.0.7713.26683, Compusense, Guelph, ON, Canada) to acquire the data.

\subsection{Statistical Analysis}

The percentage composition was calculated from the peak area data collected by SPME GC/MS analysis, and quantitative data for each compound identified in the SPME GC/MS analysis were analysed by both one- and two-way analysis of variance (ANOVA) and principal component analysis (PCA) using XLSTAT Version 2020.1.3 (Addinsoft, Paris, France). For those compounds exhibiting significant difference in the one-way ANOVA, Tukey's honest significant difference post hoc test was applied to determine which sample means differed significantly $(p<0.05)$ between geographical location and the celery genotypes. Only those compounds exhibiting significant differences between geographical location $(\mathrm{G})$, genotype $(E)$ and their interaction $(\mathrm{GxE})$ were included in the PCA.

SENPAQ version 6.3 (Qi Statistics, Kent, UK) was used to carry out the ANOVA of sensory panel data. The means from sensory data were taken over two sessions for all assessors and correlated with the percentage composition means from the instrumental data via PCA using XLSTAT.

\section{Results and Discussion}

\subsection{Volatile Composition}

In total, 118 compounds were detected in the headspace of the eight celery genotypes in both geographical locations (UK and Spain) (Table 1). Sixty-five compounds were identified in 2018 across eight genotypes, including: 22 monoterpenes, ten sesquiterpenes, eight aldehydes, five alcohols (three of which are classified as monoterpenoid 
alcohols) and five phthalides. Additional compounds were identified in the headspace of the same genotypes from the Spanish harvest including: 27 monoterpenes, 17 aldehydes, 11 sesquiterpenes and alcohols (six of which are classified as monoterpenoid alcohols), nine ketones and six phthalides. Quantitative differences were observed between the two geographical locations as well as the eight genotypes in this study, and two-way ANOVA revealed significant differences in aroma difference caused by both factors. Where Spanish grown celery displayed higher alcohol, aldehyde and ketone content, UK grown celery expressed a much higher monoterpene, sesquiterpene and phthalide content. Seventeen compounds expressed no significant difference in relative amount by these factors and seven of these came from lower boiling compounds, including camphene, sabinene and $\beta$-pinene, along with D-carvone and carvacrol. These low boiling monoterpenes were not observed to differ significantly when harvested in 2018 and 2020 in the UK [10], suggesting that monoterpenes are fundamental to the crop and factors including genotype and climate hold limited influence over the abundance of these compounds. 
Table 1. Percentage composition of volatile compounds identified in the headspace of eight celery genotypes using SPME GC/MS and harvested in UK 2018 and Spain 2019.

\begin{tabular}{|c|c|c|c|c|c|c|c|c|c|c|c|c|c|c|c|c|c|c|c|c|c|c|}
\hline \multirow{3}{*}{ Code } & \multirow{3}{*}{ Compound } & \multirow{3}{*}{$\mathrm{LRI}_{\exp }{ }^{\mathrm{A}}$} & \multirow{3}{*}{$\mathrm{ID}^{\mathrm{B}}$} & \multicolumn{16}{|c|}{ Percentage Composition (\%) $\mathrm{C}$} & \multirow{2}{*}{\multicolumn{3}{|c|}{$p$-Value $\mathrm{D}$}} \\
\hline & & & & \multicolumn{8}{|c|}{ UK } & \multicolumn{8}{|c|}{ Spain } & & & \\
\hline & & & & 5 & 8 & 10 & 12 & 15 & 18 & 22 & 25 & 5 & 8 & 10 & 12 & 15 & 18 & 22 & 25 & $\mathrm{G}^{\mathrm{E}}$ & $\mathrm{E}^{\mathrm{F}}$ & $\mathrm{GxE}^{\mathrm{G}}$ \\
\hline & Alcohols & & & & & & & & & & & & & & & & & & & & & \\
\hline $\mathrm{A} 1$ & 3-methyl-3-buten-1-ol & 730 & A & $\begin{array}{l}0.42 \pm \\
0.08 \text { abc }\end{array}$ & $\begin{array}{l}0.31 \pm \\
0.044^{\mathrm{ab}}\end{array}$ & $\begin{array}{l}0.94 \pm \\
0.27^{\mathrm{c}}\end{array}$ & $\begin{array}{l}0.35 \pm \\
0.14 \text { abc }\end{array}$ & $\begin{array}{l}0.22 \pm \\
0.07^{\mathrm{a}}\end{array}$ & $\begin{array}{l}0.23 \pm \\
0.06^{\mathrm{a}}\end{array}$ & $\begin{array}{l}0.30 \pm \\
0.12 \mathrm{ab}\end{array}$ & $\begin{array}{l}0.39 \pm \\
0.06 \mathrm{abc}\end{array}$ & $\begin{array}{l}0.60 \pm \\
0.35 \text { abc }\end{array}$ & $\begin{array}{l}0.40 \pm \\
0.06 \text { ahc }\end{array}$ & $\begin{array}{l}0.91 \pm \\
0.27^{\mathrm{bc}}\end{array}$ & $\begin{array}{l}0.59 \pm \mathrm{abc} \\
0.133^{\mathrm{abc}}\end{array}$ & $\begin{array}{l}0.36 \pm \\
0.05 \mathrm{abcc}\end{array}$ & $\begin{array}{l}0.57 \pm \\
0.22 \text { abc }\end{array}$ & $\begin{array}{l}0.54 \pm \\
0.02 \text { abc }\end{array}$ & $\begin{array}{l}0.49 \pm \\
0.13 \text { abc }\end{array}$ & $* *$ & $* *$ & $*$ \\
\hline A2 & 2-methyl-1-butanol & 742 & A & $\mathrm{nd}^{\mathrm{a}}$ & $\mathrm{nd}^{\mathrm{a}}$ & $\mathrm{nd}^{\mathrm{a}}$ & $\mathrm{nd}^{\mathrm{a}}$ & $\mathrm{nd}^{\mathrm{a}}$ & $\mathrm{nd}^{\mathrm{a}}$ & $\mathrm{nd}^{\mathrm{a}}$ & $\mathrm{nd}^{\mathrm{a}}$ & $\begin{array}{l}0.10 \pm \\
0.01 \mathrm{ab}\end{array}$ & $\begin{array}{l}0.10 \pm \\
0.03 \mathrm{ab}\end{array}$ & $\begin{array}{l}0.12 \pm \\
0.02^{\mathrm{b}}\end{array}$ & $\begin{array}{l}0.11 \pm \\
0.01 \mathrm{ab}\end{array}$ & $\mathrm{nd}^{\mathrm{a}}$ & $\begin{array}{l}0.10 \pm \mathrm{A} \\
0.04 \mathrm{ab}\end{array}$ & $\begin{array}{l}0.10 \pm \\
0.05 \mathrm{ab}\end{array}$ & $\begin{array}{l}0.10 \pm \\
0.02 \mathrm{ab}\end{array}$ & $* * *$ & $* * *$ & $* * *$ \\
\hline A3 & (E)-2-penten-1-ol & 758 & A & $\begin{array}{l}0.73 \pm \mathrm{A} \\
0.28 \mathrm{ab}\end{array}$ & $\begin{array}{l}0.42 \text { 到 } \\
0.16 \mathrm{ab}\end{array}$ & 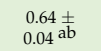 & $\begin{array}{l}0.23 \pm \\
0.08^{\mathrm{a}}\end{array}$ & $\begin{array}{l}0.32 \text { 高 } \\
0.09 \mathrm{ab}\end{array}$ & $\begin{array}{l}0.65 \mathrm{~A} \\
0.23 \mathrm{ab}\end{array}$ & $\begin{array}{l}1.2 \pm \\
0.54 \mathrm{ab}\end{array}$ & $\begin{array}{l}0.50 \pm \\
0.22 \mathrm{ab}\end{array}$ & $\begin{array}{l}0.72 \pm \\
0.34 \text { ab }\end{array}$ & $\begin{array}{l}1.3 \pm \\
0.25^{\mathrm{b}}\end{array}$ & $\begin{array}{l}1.1 . \pm \\
0.18 \mathrm{ab}\end{array}$ & $\begin{array}{l}0.71 \mathrm{a} \\
0.09 \mathrm{ab}\end{array}$ & $\begin{array}{l}0.60 \mathrm{~A} \\
0.09 \mathrm{ab}\end{array}$ & $\begin{array}{l}0.81 \mathrm{a} \\
0.31 \mathrm{ab}\end{array}$ & $\begin{array}{l}0.87 \pm \\
0.24 \text { ab }\end{array}$ & $\begin{array}{l}0.52 \pm \\
0.06 \text { ab }\end{array}$ & $* *$ & * & * \\
\hline A4 & 1-pentanol & 763 & A & $\begin{array}{l}0.28 \mathrm{at} \\
0.06^{\mathrm{a}}\end{array}$ & $\begin{array}{l}0.16 \mathrm{a} \\
0.04^{\mathrm{a}} \\
0.0\end{array}$ & $\begin{array}{l}0.31 \pm \\
0.20^{\mathrm{a}}\end{array}$ & $\begin{array}{l}0.08 \mathrm{a} \\
0.13 \pm \\
0.10^{\mathrm{a}}\end{array}$ & $\begin{array}{l}0.09 \mathrm{a} \\
0.235^{\mathrm{a}}\end{array}$ & $\begin{array}{l}0.39 \pm \\
0.14 \mathrm{ab}\end{array}$ & $\begin{array}{l}0.63 \pm \\
0.25 \mathrm{ab}\end{array}$ & $\begin{array}{l}0.22 \mathrm{at} \\
0.288^{\mathrm{a}}\end{array}$ & $\begin{array}{l}1.54 \\
1.6 \pm \\
0.27 \mathrm{~b}\end{array}$ & $\begin{array}{l}0.50 \pm \\
0.11 \mathrm{a}\end{array}$ & $\begin{array}{l}0.76 \pm \\
0.28 \mathrm{ab}\end{array}$ & $\begin{array}{l}0.49 \pm \\
0.06 \mathrm{a}\end{array}$ & $\begin{array}{l}1.11 \\
0.13 \mathrm{ab}\end{array}$ & $\begin{array}{l}0.31 \\
0.87 \\
0.34 \mathrm{ab}\end{array}$ & $\begin{array}{l}1.24 \\
1.5 \\
0.51^{\mathrm{b}}\end{array}$ & $\begin{array}{l}0.08 \\
0.22 \mathrm{ab} \\
0.22\end{array}$ & $* * * *$ & $* * *$ & $* * *$ \\
\hline \multirow[t]{2}{*}{ A5 } & 1-hexanol & 862 & A & $\begin{array}{l}0.00^{a} \\
\text { nd }\end{array}$ & $\begin{array}{l}0.04^{a} \\
n d^{a}\end{array}$ & nd ${ }^{a}$ & $\mathrm{nd}^{\mathrm{a}}$ & $\mathrm{nd}^{\mathrm{a}}$ & $\mathrm{nd}^{\mathrm{a}}$ & $\begin{array}{l}0.25^{\text {av }} \\
\text { nd }^{a}\end{array}$ & $\begin{array}{l}0.08^{a} \\
n^{a}\end{array}$ & $\begin{array}{l}0.27 \mathrm{O}^{\circ} \\
0.53 \mathrm{ab}\end{array}$ & $\begin{array}{l}0.11^{\mathrm{a}} \\
0.44 \mathrm{a} \\
027 \mathrm{ab}\end{array}$ & $\begin{array}{l}0.28 \mathrm{av} \\
0.79 \mathrm{y}\end{array}$ & $\begin{array}{l}0.06 \mathrm{a} \\
0.40 \pm\end{array}$ & $\begin{array}{l}0.1 .133 \\
0.33 \\
0.06\end{array}$ & $\begin{array}{l}0.34 \mathrm{av} \\
0.40 \mathrm{ab}\end{array}$ & $\begin{array}{l}0.50^{\circ} \\
0.48 \text { ab }\end{array}$ & $\begin{array}{l}0.22 \mathrm{av} \\
0.47 \\
0.2 \mathrm{ab}\end{array}$ & $* * *$ & $* * *$ & $* * *$ \\
\hline & $\begin{array}{c}\text { Total } \\
\text { Aldehydes }\end{array}$ & & & 1.4 & 0.84 & 1.9 & 0.71 & 0.77 & 1.3 & 2.1 & 1.2 & $\begin{array}{l}0.19 \mathrm{av} \\
3.5\end{array}$ & $\begin{array}{l}0.27 .7 \\
2.7\end{array}$ & $\begin{array}{l}0.44^{\circ} \\
3.7^{\circ}\end{array}$ & $\begin{array}{l}0.21 \mathrm{ab} \\
2.3\end{array}$ & $\begin{array}{l}0.08 \mathrm{ao} \\
2.4\end{array}$ & $\begin{array}{l}0.10^{\mathrm{aov}} \\
2.7\end{array}$ & $\frac{0.14 .5}{3.5}$ & $\begin{array}{l}0.23 \mathrm{ao} \\
2.5\end{array}$ & & & \\
\hline AH1 & 2-methyl-2-butenal & 739 & A & $\mathrm{nd}^{\mathrm{a}}$ & nd $^{\text {a }}$ & 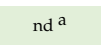 & $\mathrm{nd}^{\mathrm{a}}$ & $\mathrm{nd}^{\mathrm{a}}$ & $\mathrm{nd}^{\mathrm{a}}$ & $\mathrm{nd}^{\mathrm{a}}$ & $\mathrm{nd}^{\mathrm{a}}$ & $\begin{array}{l}0.16 \pm \\
0.0 \mathrm{bc}\end{array}$ & $\begin{array}{l}0.15 \pm \\
0.08 c\end{array}$ & $\begin{array}{l}0.14 \pm \\
0.6 \mathrm{bc}\end{array}$ & $\begin{array}{l}0.13 \pm \\
0.2 a^{2 b c}\end{array}$ & $0.23 \pm$ & $0.19 \pm$ & $0.19 \pm$ & $0.10 \pm$ & $* * *$ & $* * *$ & $* * *$ \\
\hline AH2 & (E)-2-pentenal & 753 & A & $\mathrm{nd}^{\mathrm{a}}$ & $\mathrm{nd}^{\mathrm{a}}$ & $\mathrm{nd}^{\mathrm{a}}$ & $\mathrm{nd}^{\mathrm{a}}$ & $\mathrm{nd}^{\mathrm{a}}$ & $\mathrm{nd}^{\mathrm{a}}$ & $\mathrm{nd}^{\mathrm{a}}$ & $\mathrm{nd}^{\mathrm{a}}$ & $\begin{array}{l}0.78 \pm \\
0.04 \mathrm{c}\end{array}$ & $\begin{array}{l}0.00 \pm \\
0.08^{\mathrm{a}}\end{array}$ & $\begin{array}{l}0.34 \pm \\
0.14 \mathrm{ab}\end{array}$ & $\mathrm{nd}^{\mathrm{a}}$ & $\begin{array}{l}0.78 \pm \\
0.08^{c} \\
0.03\end{array}$ & $\begin{array}{l}.0 .80^{\circ} \\
0.36^{\mathrm{c}}\end{array}$ & $\begin{array}{l}0.057 \\
0.77 \pm \\
0.09 \mathrm{bc}\end{array}$ & $\begin{array}{c}0.38 \\
0.11 \mathrm{abc} \\
0.11\end{array}$ & $* * *$ & $* * *$ & $* * *$ \\
\hline АН 3 & hexanal & 800 & A & $\begin{array}{l}9.7 \pm \\
0.8^{\mathrm{a}}\end{array}$ & $\begin{array}{l}1.3 \pm \\
0.46^{\mathrm{a}}\end{array}$ & $\begin{array}{l}2.6 \pm \\
0.32^{\mathrm{a}}\end{array}$ & $\begin{array}{l}0.65 \pm \\
0.29 \mathrm{a}\end{array}$ & $\begin{array}{l}2.0 \pm \\
0.39^{\mathrm{a}}\end{array}$ & $\begin{array}{l}8.9 \pm \\
2.7^{\mathrm{a}}\end{array}$ & $\begin{array}{l}13 \pm \\
5.5^{\mathrm{a}}\end{array}$ & $\begin{array}{l}6.3 \pm \\
1.2^{\mathrm{a}}\end{array}$ & $\begin{array}{l}25 \pm \\
7.8^{\mathrm{a}}\end{array}$ & $\begin{array}{l}24 \pm \\
6.2^{\mathrm{a}}\end{array}$ & $\begin{array}{l}14 \pm \\
5.2^{\mathrm{a}}\end{array}$ & $\begin{array}{l}8.6 \pm \\
3.6^{\mathrm{a}}\end{array}$ & $\begin{array}{l}22 \pm \\
7.5^{\mathrm{a}}\end{array}$ & $\begin{array}{l}24 \pm \\
4.9^{\mathrm{a}}\end{array}$ & $\begin{array}{l}25 \pm \\
7.0^{\mathrm{a}}\end{array}$ & $\begin{array}{l}22 \pm \\
6.3^{\mathrm{a}}\end{array}$ & $* *$ & $* *$ & $* *$ \\
\hline AH4 & (E)-2-hexenal & 849 & A & $\begin{array}{c}0.18 \pm \\
0.11 \text { abc } \\
0.11\end{array}$ & $\begin{array}{l}0.46{ }^{\circ} \\
\text { tr } \\
0.02 \mathrm{a}\end{array}$ & $\begin{array}{l}\text { tr } \\
\text { tr. } \\
0.02^{\mathrm{a}}\end{array}$ & $\begin{array}{l}0.29 \\
0.04 \mathrm{ab} \\
0.01 \mathrm{ab}\end{array}$ & $\begin{array}{l}0.03 \pm \\
0.03 \mathrm{a}\end{array}$ & $\begin{array}{l}0.15 \pm \\
0.11 \text { abc }\end{array}$ & $\begin{array}{c}5.20 \pm \\
0.02 \mathrm{abc} \\
0.08 \mathrm{ac}\end{array}$ & $\begin{array}{l}1.11 \pm \\
0.05 \mathrm{abc}\end{array}$ & $\begin{array}{l}0.86 \pm \\
0.13 \mathrm{c}\end{array}$ & $\begin{array}{l}6.24 \pm \\
0.57 \pm \\
0.24^{c}\end{array}$ & $\begin{array}{l}5.24 \\
0.30 \pm \\
0.10 \mathrm{abc}\end{array}$ & $\begin{array}{c}3.30 \pm \\
0.30 \pm \\
0.07 \mathrm{abc}\end{array}$ & $\begin{array}{l}0.55 \pm \\
0.11^{c}\end{array}$ & $\begin{array}{l}4.9{ }^{c} \pm \\
0.54 \mathrm{c} \\
0.19 \mathrm{c}\end{array}$ & $\begin{array}{l}.0 .07 \pm \\
0.575^{c}\end{array}$ & $\begin{array}{l}6.301 \pm \\
0.51 \pm \\
020\end{array}$ & $* * *$ & $* * *$ & $* * *$ \\
\hline AH5 & heptanal & 901 & A & $\begin{array}{c}0.11 \mathrm{a} \\
\operatorname{tr} \pm \\
0.03 \mathrm{ab}\end{array}$ & $n^{a}{ }^{a}$ & $\begin{array}{l}.0 .28 \\
0.28 \\
0.15\end{array}$ & $\begin{array}{l}0.16 \pm \\
0.13 \mathrm{ab}\end{array}$ & $\begin{array}{l}0.03 \\
0.25 \\
0.16 \mathrm{ab}\end{array}$ & $\begin{array}{l}0.23 \pm \\
0.14 \mathrm{ab}\end{array}$ & $\begin{array}{l}0.089 \pm \\
0.29 \mathrm{ab} \\
0.8 \mathrm{ab}\end{array}$ & $\begin{array}{l}0.25 \pm \\
0.25 \mathrm{ab}\end{array}$ & $\begin{array}{l}0.13 \mathrm{z} \\
0.68 \pm \\
0.18 \mathrm{~b}\end{array}$ & $\begin{array}{l}0.24{ }^{-} \\
0.58 \text { ab } \\
0.18 \mathrm{ab}\end{array}$ & $\begin{array}{l}0.101 \pm \\
0.13 \mathrm{ab} \\
0\end{array}$ & $\begin{array}{l}0.48 \pm \\
0.10 \mathrm{ab}\end{array}$ & $\begin{array}{l}0.112 \\
0.39 \\
0.35 \mathrm{ab}\end{array}$ & $\begin{array}{l}0.19 \\
0.57 \\
0.13 \mathrm{ab}\end{array}$ & $\begin{array}{l}0.15 \\
0.61 \pm \\
0.20 \mathrm{ab}\end{array}$ & $\begin{array}{l}0.20 \mathrm{c} \\
0.72 \pm \\
0.12^{\mathrm{b}}\end{array}$ & $* *$ & $* *$ & $* *$ \\
\hline AH6 & (E)-2-heptenal & 954 & A & $\begin{array}{l}0.19 \pm \\
0.22^{\mathrm{a}}\end{array}$ & $\begin{array}{l}1.6 \pm \\
0.55 \mathrm{ab}\end{array}$ & $\begin{array}{l}1.6 \pm \\
0.23 \mathrm{ab}\end{array}$ & $\begin{array}{l}0.52 \pm \\
0.04 \text { a }\end{array}$ & $\begin{array}{l}1.5 \pm \\
0.10 \mathrm{ab}\end{array}$ & $\begin{array}{l}3.2+ \\
1.5 \mathrm{abc}\end{array}$ & $\begin{array}{l}4.2 \pm \\
1.3 \mathrm{abc}\end{array}$ & $\begin{array}{l}1.8 \pm \\
0.97 \mathrm{ab}\end{array}$ & $\begin{array}{l}6.4 \pm \\
0.75 \mathrm{bcd}\end{array}$ & $\begin{array}{l}8.1 \pm \\
0.23 \mathrm{~cd}\end{array}$ & $\begin{array}{l}6.0 \pm \\
0.36 \mathrm{bcd}\end{array}$ & $\begin{array}{l}6.11 \pm \\
0.64 \mathrm{bcd}\end{array}$ & $\begin{array}{l}11 \pm \\
0.55^{\mathrm{d}}\end{array}$ & $\begin{array}{l}7.8 \pm \\
0.33 \mathrm{~cd}\end{array}$ & $\begin{array}{l}7.3 \pm \\
0.45 \mathrm{~cd}\end{array}$ & $\begin{array}{l}7.5 \pm \\
0.40 \mathrm{~cd}\end{array}$ & $* * *$ & $* * *$ & $* * *$ \\
\hline $\mathrm{AH7}$ & benzaldehyde & 969 & A & $\mathrm{nd}^{\mathrm{a}}$ & $\mathrm{nd}^{\mathrm{a}}$ & $\mathrm{nd}^{\mathrm{a}}$ & $\mathrm{nd}^{\mathrm{a}}$ & $\mathrm{nd}^{\mathrm{a}}$ & $\mathrm{nd}^{\mathrm{a}}$ & $\mathrm{nd}^{\mathrm{a}}$ & $\mathrm{nd}^{\mathrm{a}}$ & $\begin{array}{l}3.3 \mathrm{t} \\
18^{\mathrm{b}}\end{array}$ & $\begin{array}{l}1.7 \mathrm{~A} \\
0.50 \mathrm{ab}\end{array}$ & $1.9 \pm$ & $1.9 \pm$ & $\begin{array}{l}1.7 \pm \\
010 \mathrm{ab}\end{array}$ & $\begin{array}{l}0.38 \\
1.6 \pm \\
0.4 \mathrm{ab}\end{array}$ & $\begin{array}{l}0.45 \\
1.7 \\
02 \text { ab }\end{array}$ & $\begin{array}{l}0.40 \mathrm{c} \\
1.9 \pm \\
020^{\mathrm{b}}\end{array}$ & $* * *$ & $* * *$ & $* * *$ \\
\hline AH8 & $n$-octanal & 1007 & A & $\begin{array}{l}0.10 \pm \\
0.10 \mathrm{ab}\end{array}$ & $\mathrm{nd}^{\mathrm{a}}$ & $\begin{array}{c}0.49 \pm \\
0.06 \mathrm{abcd}\end{array}$ & $\begin{array}{l}0.27 \pm \\
0.06 \mathrm{abc}\end{array}$ & $\begin{array}{c}0.39 \pm \\
0.19 \mathrm{abcd}\end{array}$ & $\begin{array}{c}0.51 \pm \\
0.26 \mathrm{abcd}\end{array}$ & $\begin{array}{l}0.51 \pm \\
0.17 \mathrm{abcd}\end{array}$ & $\begin{array}{c}0.51 \pm \\
0.23 \text { abcd }\end{array}$ & $\begin{array}{l}1.86 \pm \\
0.19 \text { cd } \\
0.0\end{array}$ & $\begin{array}{l}0.95 \pm \\
0.22 \text { cde }\end{array}$ & $\begin{array}{c}0.56 \pm \\
0.10 \text { abcd }\end{array}$ & $\begin{array}{c}0.63 \pm \\
0.13 \mathrm{abcd}\end{array}$ & $\begin{array}{l}1.6 \pm \\
0.35 \mathrm{e}\end{array}$ & $\begin{array}{l}0.78 \pm \\
0.21 \text { bed }\end{array}$ & $\begin{array}{l}0.54 \pm \\
0.04 \text { abcd }\end{array}$ & $\begin{array}{l}1.2 . \\
0.22 \mathrm{de}\end{array}$ & $* * *$ & $* * *$ & $* * *$ \\
\hline Ан9 & phenacetaldehyde & 1049 & A & $\mathrm{nd}^{\mathrm{a}}$ & nd $^{\text {a }}$ & $\mathrm{nd}^{\mathrm{a}}$ & $\mathrm{nd}^{\mathrm{a}}$ & nd a $^{\text {a }}$ & $\mathrm{nd}^{\mathrm{a}}$ & nd $^{a}$ & $\mathrm{nd}^{\mathrm{a}}$ & $\begin{array}{l}0.31 \pm \\
0.13 \text { bc }\end{array}$ & $\begin{array}{l}0.24 \pm \\
0.04 \text { bc }\end{array}$ & $\begin{array}{l}0.26 \pm \\
0.06^{\mathrm{bc}}\end{array}$ & $\begin{array}{r}0.1 .42 \pm \\
0.06^{\mathrm{c}}\end{array}$ & $\begin{array}{l}0.26 \mathrm{t} \\
0.02 \mathrm{bc}\end{array}$ & $\begin{array}{l}0.24 \pm \\
0.06 \text { bc }\end{array}$ & $\begin{array}{l}0.23 \pm \\
0.98^{\mathrm{b}}\end{array}$ & $\begin{array}{l}0.29 \pm \\
0.05^{\mathrm{bc}}\end{array}$ & $* * *$ & $* * *$ & $* * *$ \\
\hline AH11 & $m$-tolualdehyde & 1086 & $\mathrm{~B}[18]$ & $\begin{array}{l}0.33 \pm \\
0.07 \mathrm{a}\end{array}$ & $\begin{array}{l}0.24 \pm \\
0.02^{\mathrm{a}}\end{array}$ & $\begin{array}{l}4.0 \pm \\
0.28^{c}\end{array}$ & $\begin{array}{l}1.1 \pm \\
0.28 \mathrm{ab}\end{array}$ & $\begin{array}{l}0.95 \mathrm{~A} \\
0.02 \mathrm{ab}\end{array}$ & $\begin{array}{l}0.19 \pm \\
0.02 \mathrm{a}\end{array}$ & $0.26 \pm$ & $\begin{array}{l}1.6 \pm \\
0.29^{\mathrm{b}}\end{array}$ & $\begin{array}{l}1.3 \mathrm{v} \\
0.72 \mathrm{ab} \\
0.05 \mathrm{ab}\end{array}$ & $\begin{array}{l}1.5 \mathrm{a} \\
0.66 \mathrm{ab} \\
0.26 \mathrm{ab}\end{array}$ & $\begin{array}{l}0.71 \pm \\
0.17 \mathrm{ab}\end{array}$ & $\begin{array}{l}0.91 \\
0.91 \pm \\
0.19 \mathrm{ab}\end{array}$ & $\begin{array}{l}0.89 \\
0.64 \pm \\
0.06 \mathrm{ab}\end{array}$ & $\begin{array}{l}1.2 \mathrm{z} \\
0.68 \mathrm{a} \\
0.02 \mathrm{ab}\end{array}$ & $\begin{array}{l}0.96 \mathrm{c} \\
0.57 \pm \\
0.10^{\mathrm{a}}\end{array}$ & $\begin{array}{l}1.07 \\
0.97 \pm \\
0.09 \mathrm{ab}\end{array}$ & $* * *$ & $* * *$ & $* * *$ \\
\hline AH12 & nonanal & 1105 & A & $\begin{array}{l}0.33 \pm \\
0.14 \mathrm{abc}\end{array}$ & $\begin{array}{l}0.12 \pm \\
0.02 \mathrm{ab}\end{array}$ & $\begin{array}{l}0.20 \pm \\
0.03 \mathrm{abc} \\
0.0\end{array}$ & $\begin{array}{l}0.10 \pm \\
0.01 \mathrm{a}\end{array}$ & $\begin{array}{l}0.17 \pm \\
0.03 \text { abc }\end{array}$ & $\begin{array}{l}0.16 \pm \\
0.10 \mathrm{abc}\end{array}$ & $\begin{array}{l}0.22 \pm \\
0.17 \text { abc }\end{array}$ & $\begin{array}{l}0.19 \pm \\
0.09 \text { abc }\end{array}$ & $\begin{array}{l}0.68 \pm \\
0.111^{c}\end{array}$ & $\begin{array}{l}0.59 \pm \\
0.18 \mathrm{abc}\end{array}$ & $\begin{array}{l}0.39 \pm \\
0.10^{\mathrm{b}}\end{array}$ & $\begin{array}{l}0.35 \pm \\
0.13 \text { abc }\end{array}$ & $\begin{array}{l}0.57 \pm \\
0.16 \mathrm{abc}\end{array}$ & $\begin{array}{l}0.64 \pm \\
0.35 \text { bc }\end{array}$ & $\begin{array}{l}0.61 \pm \\
0.08 \text { abc } \\
0.04\end{array}$ & $\begin{array}{l}0.59 \pm \\
0.11 \text { abc }\end{array}$ & $* * *$ & $* * *$ & $* * *$ \\
\hline AH13 & $(E, E)-2,4$-octadienal & 1110 & A & $\mathrm{nd}^{\mathrm{a}}$ & $\mathrm{nd}^{\mathrm{a}}$ & nd $^{\text {a }}$ & $\mathrm{nd}^{\mathrm{a}}$ & $\mathrm{nd}^{\mathrm{a}}$ & $\mathrm{nd}^{\mathrm{a}}$ & $\mathrm{nd}^{\mathrm{a}}$ & $\mathrm{nd}^{\mathrm{a}}$ & $\begin{array}{l}0.11 \\
0.15 \pm \\
0.05\end{array}$ & $\begin{array}{l}0.13 \pm \\
0.13 \\
0.54\end{array}$ & $\begin{array}{l}0.10^{\circ} \\
0.11 \mathrm{~b}\end{array}$ & $\begin{array}{l}0.13 \pm \\
0.33 \mathrm{~b}\end{array}$ & $\begin{array}{l}0.16 \pm \\
0.16 b^{b}\end{array}$ & $\begin{array}{l}0.15 \pm \\
0.03 \mathrm{~b} \\
0.03\end{array}$ & $\begin{array}{l}0.14 \pm \\
0.55\end{array}$ & $\begin{array}{l}0.20 \pm \\
0.02 \mathrm{~b}\end{array}$ & $* * *$ & $* * *$ & $* * *$ \\
\hline AH14 & $(E, Z)-2,6$-nonadienal & 1162 & A & $\mathrm{nd}^{\mathrm{a}}$ & nd a & $\mathrm{nd}^{\mathrm{a}}$ & $\mathrm{nd}^{\mathrm{a}}$ & $\mathrm{nd}^{\mathrm{a}}$ & $\mathrm{nd}^{\mathrm{a}}$ & $\mathrm{nd}^{\mathrm{a}}$ & $\mathrm{nd}^{\mathrm{a}}$ & $\begin{array}{l}0.05 \\
0.10 \pm \\
0.06 \mathrm{ab}\end{array}$ & $\begin{array}{l}0.04 \\
0.15 \\
0.03 \text { abc }\end{array}$ & $\begin{array}{c}0.01 \\
0.11 \\
0.02 \mathrm{abc}\end{array}$ & $\begin{array}{l}0.03 \\
0.12 \pm \\
0.02 \text { abc }\end{array}$ & $\begin{array}{l}0.029 \pm \\
0.29 \pm \\
0.10^{c}\end{array}$ & $\begin{array}{l}0.03 \\
0.23 \pm \\
0.20 \mathrm{bc}\end{array}$ & $\begin{array}{l}0.03 \pm \\
0.23 \pm \\
0.16 \mathrm{bc}\end{array}$ & 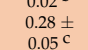 & $* * *$ & $* * *$ & $* * *$ \\
\hline AH15 & (E)-2-nonenal & 1165 & A & $\mathrm{nd}^{\mathrm{a}}$ & $\mathrm{nd}^{\mathrm{a}}$ & $\mathrm{nd}^{\mathrm{a}}$ & $\mathrm{nd}^{\mathrm{a}}$ & $\mathrm{nd}^{\mathrm{a}}$ & $\mathrm{nd}^{\mathrm{a}}$ & $\mathrm{nd}^{\mathrm{a}}$ & $\mathrm{nd}^{\mathrm{a}}$ & $\begin{array}{l}0.10 \pm \\
0.03 \mathrm{ab}\end{array}$ & $\begin{array}{l}0.10 \pm \\
0.102 \mathrm{ab}\end{array}$ & $\begin{array}{l}\operatorname{tr} \pm \\
0.03 \mathrm{ab}\end{array}$ & $\begin{array}{l}0.14 \mathrm{I} \\
0.02 \mathrm{~b}\end{array}$ & $\begin{array}{l}0.10 \mathrm{a} \\
0.01 \mathrm{ab} \\
0.01\end{array}$ & $\begin{array}{l}0.10 \pm \\
0.101 \mathrm{ab}\end{array}$ & $\begin{array}{l}\text { tr } \\
0.05 \mathrm{ab}\end{array}$ & $\begin{array}{l}0.12 \pm \\
0.10^{\mathrm{b}}\end{array}$ & $* * *$ & $* * *$ & $* * *$ \\
\hline AH16 & myrtenal & 1207 & B [19] & $\mathrm{nd}^{\mathrm{a}}$ & $\mathrm{nd}^{\mathrm{a}}$ & $\mathrm{nd}^{\mathrm{a}}$ & $\mathrm{nd}^{\mathrm{a}}$ & $\mathrm{nd}^{\mathrm{a}}$ & $\mathrm{nd}^{\mathrm{a}}$ & $\mathrm{nd}^{\mathrm{a}}$ & $\mathrm{nd}^{\mathrm{a}}$ & $\begin{array}{l}0.19 \\
0.19 \\
0 . \mathrm{ab}\end{array}$ & $\begin{array}{l}0.14 \pm \\
0.02 \mathrm{a}\end{array}$ & $\begin{array}{l}0.10 \pm \\
0.03 \mathrm{a}\end{array}$ & $\begin{array}{l}0.02 \\
0.11 \pm \\
0.01 \mathrm{a}\end{array}$ & $\begin{array}{l}0.16 \pm \\
0.04 \text { ab }\end{array}$ & $\begin{array}{l}0.15 \\
0.15 \\
0 . \mathrm{ab}\end{array}$ & $\begin{array}{l}0.10 \pm \\
0.06^{\mathrm{a}}\end{array}$ & $\begin{array}{l}. .107 \\
0.37 \\
0.21 \mathrm{~b}\end{array}$ & $* * *$ & $* * *$ & $* * *$ \\
\hline \multirow[t]{2}{*}{ AH17 } & $(E, E)-2,6$-nonadienal & 1156 & A & $\begin{array}{l}0.21 \mathrm{a} \\
0.04 \mathrm{ab}\end{array}$ & $\begin{array}{l}0.30 \pm \\
0.03 \text { ab }\end{array}$ & $0.18 \mathrm{ab}$ & $\begin{array}{l}0.18 \mathrm{~A} \\
0.04 \mathrm{ab}\end{array}$ & $\begin{array}{l}0.17 \pm \\
0.03 \text { ab }\end{array}$ & $\begin{array}{l}0.16 \pm \\
0.08 \mathrm{ab}\end{array}$ & $\underset{0.03^{\mathrm{a}}}{\operatorname{tr} \pm}$ & $\begin{array}{l}0.22 \pm \\
0.08 \text { ab }\end{array}$ & $\begin{array}{l}0.36 \pm \\
0.11 \mathrm{ab}\end{array}$ & $\begin{array}{l}.0 .2^{2} \\
0.48 \\
0.24\end{array}$ & $\begin{array}{l}0.033^{2} \\
0.03 \mathrm{ab}\end{array}$ & $\begin{array}{l}0.016 \pm \\
0.16 \mathrm{ab} \\
0.05\end{array}$ & $\begin{array}{l}0.41 \pm \\
0.11 \text { ab } \\
0.11\end{array}$ & $\begin{array}{l}0.35 \pm \\
0.11 \mathrm{ab}\end{array}$ & $\begin{array}{l}0.066^{\circ} \\
0.42 \mathrm{ab} \\
0.22\end{array}$ & $\begin{array}{l}0.21 \\
0.20 \pm \\
0.17^{\mathrm{ab}}\end{array}$ & * & * & * \\
\hline & $\begin{array}{l}\text { Total } \\
\text { Esters }\end{array}$ & & & 11 & & 9.4 & & & & & & 44 & $\frac{.44}{41}$ & 28 & 23 & 44 & $\frac{.11}{44}$ & 43 & 41 & & & \\
\hline E1 & methyl butanoate & 717 & A & $\begin{array}{l}\operatorname{tr} \pm \mathrm{bc} \\
0.03 \mathrm{abc}\end{array}$ & $\underset{0.01 \mathrm{a}}{\operatorname{tr} \pm}$ & $\begin{array}{l}\mathrm{tr} \pm \mathrm{bc} \\
0.02 \mathrm{abc}\end{array}$ & $\begin{array}{c}\mathrm{tr} \pm \\
0.01 \mathrm{ab}\end{array}$ & $\begin{array}{c}\mathrm{tr} \pm \mathrm{t} \\
0.02 \mathrm{ab}\end{array}$ & $\begin{array}{c}\mathrm{tr} \pm \\
0.04 \mathrm{ab}\end{array}$ & $\begin{array}{c}\mathrm{tr} \pm \\
0.0 \mathrm{ab} \\
\mathrm{ab}\end{array}$ & $\begin{array}{c}\mathrm{tr} \pm \\
0.01 \mathrm{ab}\end{array}$ & $\begin{array}{l}0.22 \pm \\
0.14 \mathrm{~cd}\end{array}$ & $\begin{array}{c}0.18 \pm \\
0.01 \mathrm{abcd}\end{array}$ & $\begin{array}{l}0.25 \pm \\
0.04{ }^{\mathrm{d}}\end{array}$ & $\begin{array}{c}0.17 \pm \\
0.01 \mathrm{abcd}\end{array}$ & $\begin{array}{c}0.18 \pm \\
0.04 \mathrm{abcd}\end{array}$ & $\begin{array}{c}0.18 \pm \\
0.04 \mathrm{abcd}\end{array}$ & $\begin{array}{c}0.16 \pm \\
0.02 \mathrm{abcd}\end{array}$ & $\begin{array}{l}0.19 \pm \\
0.03 \mathrm{bcd}\end{array}$ & $* * *$ & $* * *$ & $* * *$ \\
\hline E2 & methyl pentanoate & 837 & A & $\mathrm{nd}^{\mathrm{a}}$ & $\mathrm{nd}^{\mathrm{a}}$ & nd $^{\text {a }}$ & $\mathrm{nd}^{\mathrm{a}}$ & $\mathrm{nd}^{\mathrm{a}}$ & $\mathrm{nd}^{\mathrm{a}}$ & $\mathrm{nd}^{\mathrm{a}}$ & $\mathrm{nd}^{\mathrm{a}}$ & $\begin{array}{l}0.34 \mathrm{t} \\
0.23^{\mathrm{b}}\end{array}$ & $\begin{array}{l}0.24 \pm \\
0.22 \mathrm{ab}\end{array}$ & $\begin{array}{l}0.37 \\
0.13^{\mathrm{b}}\end{array}$ & $\begin{array}{r}0.40 \pm \\
0.09 \mathrm{~b}\end{array}$ & $\begin{array}{l}0.23 \\
0.07 \mathrm{ab}\end{array}$ & $\begin{array}{l}0.39 \pm \\
0.18\end{array}$ & $0.27 \pm$ & $\begin{array}{l}0.30 \mathrm{a} \\
0.05 \mathrm{ab}\end{array}$ & $* * *$ & $* * *$ & $* * *$ \\
\hline Е3 & methyl hexanoate & 921 & A & $\mathrm{nd}^{\mathrm{a}}$ & $\mathrm{nd}^{\mathrm{a}}$ & $\mathrm{nd}^{\mathrm{a}}$ & $\mathrm{nd}^{\mathrm{a}}$ & $\mathrm{nd}^{\mathrm{a}}$ & $\mathrm{nd}^{\mathrm{a}}$ & $\mathrm{nd}^{\mathrm{a}}$ & $\mathrm{nd}^{\mathrm{a}}$ & $\begin{array}{l}0.25 \mathrm{a} \\
0.12 \mathrm{ab}\end{array}$ & $\begin{array}{l}0.29 \pm \\
0.16 \mathrm{ab}\end{array}$ & $\begin{array}{l}0.12 \pm \\
0.01 \mathrm{ab}\end{array}$ & $\begin{array}{l}0.10 \pm \\
0.03 \mathrm{ab}\end{array}$ & $\begin{array}{l}0.25 \pm \\
0.09 \mathrm{ab}\end{array}$ & $\begin{array}{l}0.38 \pm \\
0.10^{\mathrm{b}}\end{array}$ & $\begin{array}{l}0.28 \pm \\
0.20^{\mathrm{bc}}\end{array}$ & $\begin{array}{l}0.024 \pm \\
0.211^{a b}\end{array}$ & $* * *$ & $* * *$ & $* * *$ \\
\hline E4 & carveol acetate & 1343 & B [20] & $\mathrm{nd}^{\text {a }}$ & $\mathrm{nd}^{\mathrm{a}}$ & $\mathrm{nd}^{\mathrm{a}}$ & $\mathrm{nd}^{\mathrm{a}}$ & $\mathrm{nd}^{\mathrm{a}}$ & $\mathrm{nd}^{\mathrm{a}}$ & $\mathrm{nd}^{\mathrm{a}}$ & $\mathrm{nd}^{\mathrm{a}}$ & $\begin{array}{l}0.21 \pm \\
0.21 \\
0.5 \mathrm{bc}\end{array}$ & $\begin{array}{l}0.16 \text {. } \\
0.14+ \\
0.02 \mathrm{ab}\end{array}$ & $\begin{array}{l}0.22 \pm \\
0.24 \\
0.4 c\end{array}$ & $\begin{array}{l}0.17 \pm \\
0.17 \\
0.4 \mathrm{bc}\end{array}$ & $\begin{array}{l}0.20 \pm \\
0.20 \\
0.4 c\end{array}$ & $\begin{array}{l}0.100 \\
0.27 \pm \\
0.08 \mathrm{Bc}\end{array}$ & $\begin{array}{l}0.100 \\
0.20 \pm \\
0.05\end{array}$ & $\begin{array}{l}0.29 \pm \\
0.10^{\mathrm{c}}\end{array}$ & $x * *$ & $* * *$ & $* * *$ \\
\hline \multirow[t]{2}{*}{ E5 } & hexyl isobutanoate & 1378 & B [21] & $\begin{array}{l}0.10 \pm \\
0.03\end{array}$ & $\begin{array}{c}0.10 \pm \\
0.04\end{array}$ & $\begin{array}{c}0.14 \pm \\
0.02\end{array}$ & 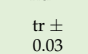 & $\begin{array}{c}0.10 \pm \\
0.05\end{array}$ & $\begin{array}{l}0.16 \pm \\
0.04\end{array}$ & $\begin{array}{l}0.32 \pm \\
0.06\end{array}$ & $\begin{array}{c}0.12 \pm \\
0.03\end{array}$ & $\begin{array}{l}0.050 \\
0.15 \pm \\
0.12\end{array}$ & $\begin{array}{l}0.02 \pm \\
0.12 \pm \\
0.12\end{array}$ & $\begin{array}{l}.0 .40 \pm \\
0.04 \\
0.04\end{array}$ & $\begin{array}{l}0.024 \pm \\
0.22 \pm \\
0.11\end{array}$ & $\begin{array}{l}0.18 \pm \\
0.18 \\
0.13\end{array}$ & $\begin{array}{l}.0 .08 \\
0.11 \pm \\
0.16\end{array}$ & $\begin{array}{l}0.05 \mathrm{a} \\
0.36 \pm \\
0.23\end{array}$ & $\begin{array}{l}0.102 \\
0.13 \pm \\
0.11\end{array}$ & ns & $\mathrm{ns}$ & ns \\
\hline & $\begin{array}{c}\text { Total } \\
\text { Ketones }\end{array}$ & & & 0.14 & 0.10 & 0.20 & 0.07 & 0.11 & 0.19 & 0.36 & 0.14 & 1.2 & $\begin{array}{l}0.12 \\
1.0\end{array}$ & $\begin{array}{l}0.04 \\
1.4\end{array}$ & $\begin{array}{l}0.11 \\
1.0\end{array}$ & $\begin{array}{l}0.13 \\
1.0\end{array}$ & 1.3 & $\begin{array}{l}0.23 \\
1.3\end{array}$ & $\begin{array}{l}0.11 \\
1.2\end{array}$ & & & \\
\hline
\end{tabular}


Table 1. Cont.

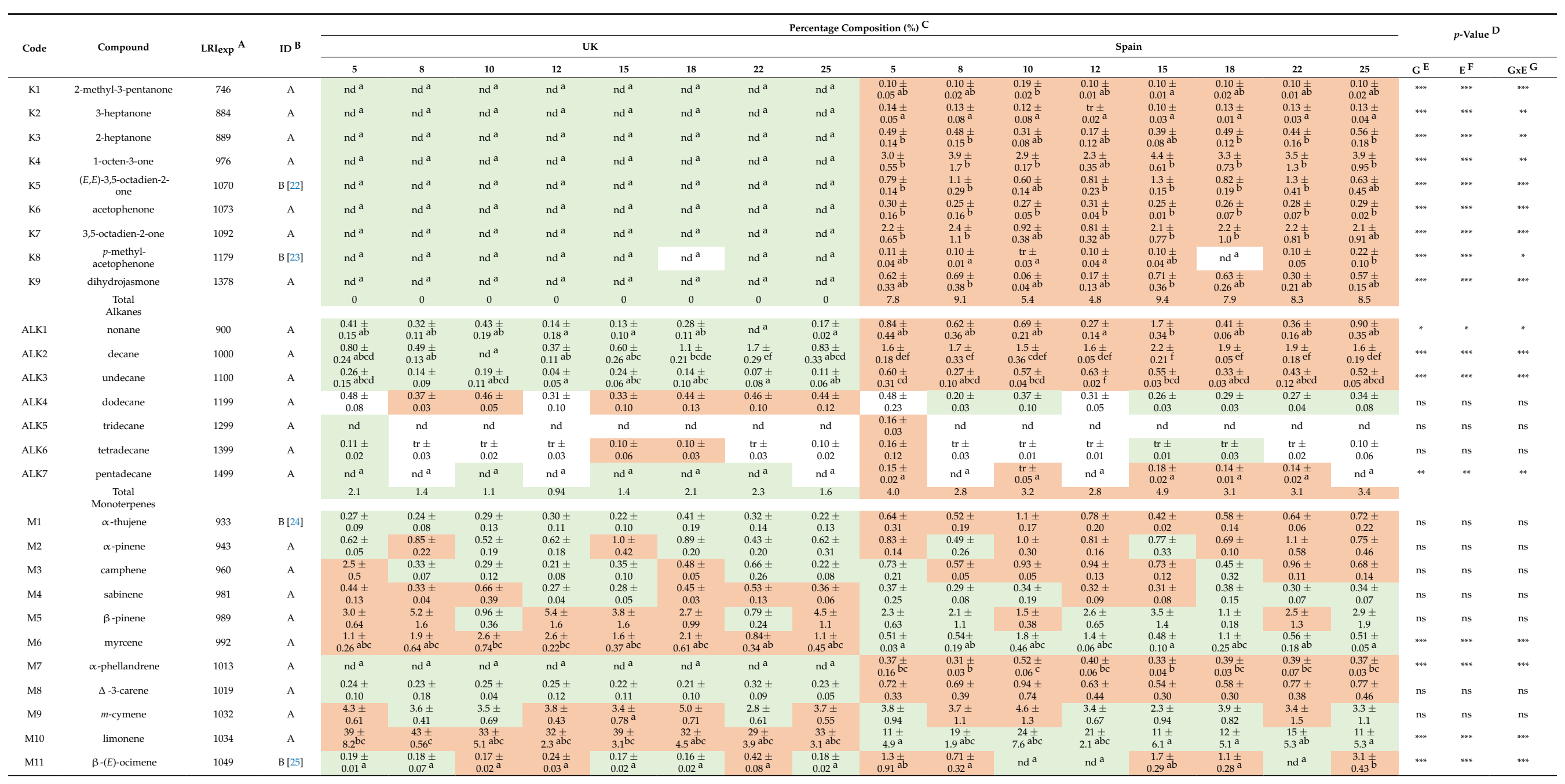


Table 1. Cont.

\begin{tabular}{|c|c|c|c|c|c|c|c|c|c|c|c|c|c|c|c|c|c|c|c|c|c|c|}
\hline \multirow{3}{*}{ Code } & \multirow{3}{*}{ Compound } & \multirow{3}{*}{ LRI $_{\exp }{ }^{A}$} & \multirow{3}{*}{$\mathrm{ID}^{\mathrm{B}}$} & \multicolumn{16}{|c|}{ Percentage Composition (\%) $\mathrm{C}$} & \multirow{2}{*}{\multicolumn{3}{|c|}{$p$-Value $\mathrm{D}$}} \\
\hline & & & & \multicolumn{8}{|c|}{ UK } & \multicolumn{8}{|c|}{ Spain } & & & \\
\hline & & & & 5 & 8 & 10 & 12 & 15 & 18 & 22 & 25 & 5 & 8 & 10 & 12 & 15 & 18 & 22 & 25 & $\mathrm{G}^{\mathrm{E}}$ & ${ }_{E} F$ & $\mathrm{GxE} G$ \\
\hline M12 & $\gamma$-terpinene & 1066 & A & $\begin{array}{l}\begin{array}{l}4.2 \pm \\
1.2^{\mathrm{bcd}}\end{array} \\
\end{array}$ & $\begin{array}{l}4.3 \pm \pm \\
1.2 \mathrm{bcd}\end{array}$ & $\begin{array}{l}3.6 \pm \frac{ \pm}{0} \\
0.60^{\mathrm{abcd}}\end{array}$ & $\begin{array}{l}5.9 \pm \mathrm{I} \\
0.28^{\mathrm{d}}\end{array}$ & $\begin{array}{l}5.6 \pm \\
0.27 \mathrm{~cd}\end{array}$ & $\begin{array}{l}5.5 \pm \\
1.4 \text { cd }\end{array}$ & $\begin{array}{l}2.1 \pm \pm \\
0.90 \mathrm{ab}\end{array}$ & 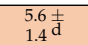 & $\begin{array}{l}0.72 \pm \\
0.12 \mathrm{a}\end{array}$ & $\begin{array}{l}2.6 \pm \\
1.4 \text { abcd }\end{array}$ & $\begin{array}{l}2.2 \pm \mathrm{abc} \\
0.36 \text { abc }\end{array}$ & $\begin{array}{l}2.0 \pm \\
0.35 \mathrm{ab}\end{array}$ & $\begin{array}{l}1.2 \pm \\
0.24 \mathrm{ab}\end{array}$ & $\begin{array}{l}1.1+ \pm \\
0.24 \mathrm{ab}\end{array}$ & $\begin{array}{l}1.1 \pm \pm \\
0.20 \mathrm{ab}\end{array}$ & $\begin{array}{l}1.1 \mathrm{t} \\
0.36 \mathrm{ab}\end{array}$ & $* * *$ & $* * *$ & $* * *$ \\
\hline M13 & terpinolene & 1097 & A & $\begin{array}{l}0.62 \pm \\
0.19 \mathrm{abc}\end{array}$ & $\begin{array}{l}0.89 \pm \\
0.07 \mathrm{c}\end{array}$ & $\begin{array}{l}0.53 \pm \\
0.09 \mathrm{abc}\end{array}$ & $\begin{array}{l}0.43 \pm \\
0.01 \mathrm{abc}\end{array}$ & $\begin{array}{l}0.36 \pm \mathrm{c} \\
0.22 \mathrm{abc}\end{array}$ & $\begin{array}{l}0.73 \pm \\
0.20 \mathrm{cc}\end{array}$ & $\begin{array}{l}0.57 \pm \\
0.14 \text { abc }\end{array}$ & $\begin{array}{l}0.90 \pm \\
0.31 \mathrm{c}\end{array}$ & $\begin{array}{l}0.35 \pm \\
0.08 \text { abc }\end{array}$ & $\begin{array}{l}0.25 \pm \\
0.18 \mathrm{abc}\end{array}$ & $\begin{array}{l}0.13 \pm \\
0.08 \text { ab }\end{array}$ & $\begin{array}{l}0.02 \mathrm{a} \\
0.14 \mathrm{ab}\end{array}$ & $\begin{array}{l}0.38 \pm \\
0.14 \mathrm{abc}\end{array}$ & $\begin{array}{l}0.34 \pm \\
0.14 \mathrm{abc}\end{array}$ & $\mathrm{nd}^{\mathrm{a}}$ & $\begin{array}{l}0.25 \pm \\
0.18 \text { abc }\end{array}$ & $* * *$ & $* * *$ & $* *$ \\
\hline M14 & allo-ocimene & 1132 & B [26] & $\begin{array}{l}0.11 \pm \\
0.06 \mathrm{ab}\end{array}$ & $\begin{array}{l}0.10 \pm \\
0.01 \text { ab } \\
0.01\end{array}$ & $\begin{array}{l}0.030 \mathrm{a} \\
0.05 \mathrm{ab}\end{array}$ & $\begin{array}{l}0.31 \pm \\
0.03 \\
0\end{array}$ & $\begin{array}{l}0.24 \pm \\
<0.01 \mathrm{ab}\end{array}$ & $\begin{array}{l}0.13 \mathrm{at} \\
0.04 \mathrm{ab}\end{array}$ & $\begin{array}{l}0.141 \pm \\
0.27^{\mathrm{b}}\end{array}$ & $\begin{array}{l}0.13 \\
0.08 \text { ab } \\
0.04\end{array}$ & nd a & nd $^{\text {a }}$ & $\mathrm{nd}^{\mathrm{a}}$ & $\mathrm{nd}^{\mathrm{a}}$ & $\mathrm{nd}^{\mathrm{a}}$ & nd $^{\text {a }}$ & $\mathrm{nd}^{\mathrm{a}}$ & $\mathrm{nd}^{\mathrm{a}}$ & $* * *$ & $* * *$ & $* *$ \\
\hline M15 & $\beta$-thujone & 1124 & B [23] & $\mathrm{nd}^{\mathrm{a}}$ & $\mathrm{nd}^{\mathrm{a}}$ & nd $^{\text {a }}$ & $\mathrm{nd}^{\mathrm{a}}$ & nd $^{a}$ & $\mathrm{nd}^{\mathrm{a}}$ & $\mathrm{nd}^{\mathrm{a}}$ & $\mathrm{nd}^{\mathrm{a}}$ & $\begin{array}{l}0.10 \pm \\
0.02 \mathrm{ab}\end{array}$ & $\begin{array}{c}\operatorname{tr} \pm \\
0.02 \mathrm{a}\end{array}$ & $\begin{array}{l}0.10 \pm \\
0.01 \text { abc }\end{array}$ & $\begin{array}{l}0.20 \pm \\
0.04^{c}\end{array}$ & $\begin{array}{c}\operatorname{tr} \pm \\
0.02 \mathrm{ab}\end{array}$ & $0.10 \pm$ & $\begin{array}{l}0.17 \pm \\
0.12 \mathrm{bc}\end{array}$ & $0.10 \pm$ & $* * *$ & $* * *$ & $* * *$ \\
\hline M16 & $p$-mentha-1,5,8-triene & 1135 & В [27] & $\begin{array}{l}0.26 \pm \\
0.05 \mathrm{ab}\end{array}$ & $\begin{array}{l}0.10 \pm \\
0.01 \mathrm{ab}\end{array}$ & $\begin{array}{l}0.22 \pm \\
0.02 \mathrm{ab}\end{array}$ & $\begin{array}{l}0.56 \frac{\mathrm{t}}{0.09} \\
0 . \mathrm{b}\end{array}$ & $\begin{array}{l}0.26 \pm \\
0.07 \mathrm{ab}\end{array}$ & $\begin{array}{l}0.13 \pm \\
0.09 \mathrm{ab}\end{array}$ & $\begin{array}{l}0.49 \pm \\
0.17 \mathrm{ab}\end{array}$ & $\begin{array}{l}0.19 \pm \\
0.08 \mathrm{ab}\end{array}$ & $\begin{array}{l}0.10 \pm \\
0.02 \mathrm{ab}\end{array}$ & $\begin{array}{l}\operatorname{tr} \pm \\
0.02 \mathrm{a}\end{array}$ & $\begin{array}{l}0.16 \pm \\
0.04 \text { ab }\end{array}$ & $\begin{array}{l}0.55 \\
0.15 \mathrm{ab}\end{array}$ & $\begin{array}{l}0.10 \pm \\
0.01 \mathrm{ab}\end{array}$ & $\begin{array}{l}0.17 \pm \\
0.05 \mathrm{ab}\end{array}$ & $\begin{array}{l}0.50 \pm \\
0.27 \mathrm{ab}\end{array}$ & $\begin{array}{l}0.10 \pm \\
0.06 \mathrm{ab}\end{array}$ & $* *$ & $* *$ & $* *$ \\
\hline M17 & (Z)-carveol & 1147 & B [19] & $\begin{array}{l}0.48 \pm \\
0.13 \mathrm{bcd}\end{array}$ & $\begin{array}{l}0.57 \text { 巟 } \\
0.17 \text { cd }\end{array}$ & $\begin{array}{l}0.23 \pm \\
0.08 \mathrm{abc}\end{array}$ & 0.18 ab & $\begin{array}{l}0.24 \pm \\
0.02 \mathrm{ab}\end{array}$ & $\begin{array}{l}0.31 \pm \mathrm{ac} \\
0.21 \mathrm{abc}\end{array}$ & $\begin{array}{c}\operatorname{tr} \pm \\
0.03 \mathrm{a}\end{array}$ & $\begin{array}{l}0.13 \\
0.10 \mathrm{ab}\end{array}$ & $\begin{array}{l}0.51 \pm \\
0.07 \mathrm{~cd}\end{array}$ & $\begin{array}{l}0.02 \\
0.21 \mathrm{bcd} \\
0.21\end{array}$ & $\begin{array}{l}0.65 \pm \\
0.09^{\mathrm{d}}\end{array}$ & $\begin{array}{l}0.44 \pm \\
0.02 \mathrm{bcd}\end{array}$ & $\begin{array}{c}0.34 \pm \\
0.07 \mathrm{abcd}\end{array}$ & $\begin{array}{l}0.051 \pm \\
0.14 \mathrm{~cd}\end{array}$ & $\begin{array}{c}0.26 \pm \\
0.02 \mathrm{abcd}\end{array}$ & $\begin{array}{l}0.06 \pm 0 \\
0.02 \frac{1}{d}\end{array}$ & $* * *$ & $* * *$ & $* * *$ \\
\hline M18 & $\begin{array}{l}\text { pentylycyclohexa-1,3- } \\
\text { diene }\end{array}$ & 1166 & B [19] & $\begin{array}{l}0.20 \pm \\
0.05 \\
a b\end{array}$ & $\begin{array}{l}0.23 \pm \\
0.08 \mathrm{ab}\end{array}$ & $\begin{array}{l}0.25 \mathrm{I} \\
0.03 \mathrm{ab}\end{array}$ & $\begin{array}{l}0.46 \pm \\
0.11 \mathrm{abc}\end{array}$ & $\begin{array}{l}0.31 \pm \\
0.03 \mathrm{ab}\end{array}$ & $\begin{array}{l}0.21 \mathrm{a} \pm \\
0.044^{\mathrm{a}}\end{array}$ & $\begin{array}{l}0.26 \pm \\
0.16 \mathrm{ab}\end{array}$ & $\begin{array}{l}0.20 \pm \\
0.01 \mathrm{ab}\end{array}$ & $\begin{array}{l}0.20 \pm \\
0.06 \mathrm{ab}\end{array}$ & $\begin{array}{l}0.13 \pm \\
0.09 \mathrm{a} \\
0.12\end{array}$ & $\begin{array}{l}0.09 \mathrm{a} \\
0.08 \mathrm{ab}\end{array}$ & $\begin{array}{l}0.20 \pm \\
0.22 \mathrm{ab}\end{array}$ & $\begin{array}{l}0.16 \pm \\
0.05 \mathrm{ab}\end{array}$ & $\begin{array}{l}0.19 \pm \\
0.02 \mathrm{ab}\end{array}$ & $\begin{array}{l}0.012 \pm \\
0.09 \mathrm{a} \\
0.09\end{array}$ & $\begin{array}{l}0.230 \pm \\
0.14 \mathrm{ab}\end{array}$ & * & * & $*$ \\
\hline M19 & (Z)-dihydrocarvone & 1208 & A & $\begin{array}{l}0.39 \pm \\
0.09 \mathrm{~b}\end{array}$ & $\begin{array}{l}0.36 \pm \\
0.05 \mathrm{~b}\end{array}$ & $\begin{array}{l}0.35 \mathrm{t} \\
0.08^{\mathrm{b}}\end{array}$ & $\begin{array}{l}0.19 \pm \\
0.06 \mathrm{ab}\end{array}$ & $\begin{array}{l}0.27 \mathrm{z} \\
0.05 \mathrm{ab}\end{array}$ & $\begin{array}{l}0.18 \mathrm{ab} \\
0.04 \mathrm{ab}\end{array}$ & $\begin{array}{l}0.20 \pm \\
0.08 \mathrm{ab}\end{array}$ & $\begin{array}{l}0.26 \pm \\
0.02 \text { ab }\end{array}$ & $\begin{array}{l}0.00 \mathrm{~s} \\
0.035 \\
0.03 \mathrm{~b}\end{array}$ & $\begin{array}{l}0.28 \mathrm{~J} \\
0.02 \mathrm{ab}\end{array}$ & $\begin{array}{l}0.00 \mathrm{O} \\
0.00^{\mathrm{b}} \mathrm{b}\end{array}$ & $\begin{array}{l}0.02 \mathrm{at} \\
0.06 \mathrm{ab}\end{array}$ & $\begin{array}{l}0.23 \mathrm{a} \\
0.12 \mathrm{ab}\end{array}$ & $\begin{array}{l}0.20 \pm \\
0.14 \text { ab }\end{array}$ & $\mathrm{nd}^{\mathrm{a}}$ & $\begin{array}{l}0.14 \\
0.39 \\
0.06 \mathrm{~b}\end{array}$ & $* *$ & $* *$ & $* *$ \\
\hline м20 & camphor & 1157 & A & $\mathrm{nd}^{\mathrm{a}}$ & $\mathrm{nd}^{\mathrm{a}}$ & $\mathrm{nd}^{\mathrm{a}}$ & $\mathrm{nd}^{\mathrm{a}}$ & $\mathrm{nd}^{\mathrm{a}}$ & $\mathrm{nd}^{\mathrm{a}}$ & $\mathrm{nd}^{\mathrm{a}}$ & $\mathrm{nd}^{\mathrm{a}}$ & $\begin{array}{l}0.27 \pm \\
0.15 \mathrm{bc}\end{array}$ & $\begin{array}{l}0.17 \pm \\
0.04 \text { abc }\end{array}$ & $\begin{array}{l}0.22 \pm \\
0.06 \text { abc }\end{array}$ & $\begin{array}{l}0.17 \pm \\
0.05 \text { abc }\end{array}$ & $\begin{array}{l}0.18 \pm \\
0.08 \text { abc }\end{array}$ & $\begin{array}{l}0.23 \pm \\
0.06 \mathrm{bc}\end{array}$ & $\begin{array}{l}0.15 \pm \\
0.03 \mathrm{ab}\end{array}$ & $\begin{array}{l}0.38 \pm \\
0.13^{\mathrm{c}}\end{array}$ & $* * *$ & $* * *$ & $* * *$ \\
\hline м21 & isoborneol & 1173 & A & $\mathrm{nd}^{\mathrm{a}}$ & $\mathrm{nd}^{\mathrm{a}}$ & $\mathrm{nd}^{\mathrm{a}}$ & $\mathrm{nd}^{\mathrm{a}}$ & $\mathrm{nd}^{\mathrm{a}}$ & $\mathrm{nd}^{\mathrm{a}}$ & $\mathrm{nd}^{\mathrm{a}}$ & $\mathrm{nd}^{\mathrm{a}}$ & $\begin{array}{l}0.13 \\
0.25 \\
0.14^{\mathrm{b}}\end{array}$ & $\begin{array}{l}0.04 \text { - } \\
0.03 \mathrm{ab}\end{array}$ & $\begin{array}{l}0.06 \mathrm{c} \\
0.06 \mathrm{ab}\end{array}$ & $\begin{array}{l}0.05 \mathrm{a} \\
0.04 \mathrm{ab} \\
0.04\end{array}$ & $\begin{array}{l}0.19= \\
0.04 \mathrm{ab}\end{array}$ & $\begin{array}{l}0.00- \\
0.04 \\
0.04\end{array}$ & $\begin{array}{l}0.18 \pm \\
0.05 \text { ab }\end{array}$ & $\begin{array}{l}0.23 \\
0.12 \pm \\
0.12 \mathrm{~b}\end{array}$ & $* * *$ & $* * *$ & $* * *$ \\
\hline M22 & (E)-dihydrocarvone & 1240 & В [27] & $\begin{array}{l}0.79 \pm \frac{f}{} \\
0.12^{f}\end{array}$ & $\begin{array}{l}0.79 \pm \frac{\mathrm{f}}{0.14^{\mathrm{f}}} \\
{ }^{2}\end{array}$ & $\begin{array}{l}0.67 \pm \\
0.10 \text { ef }\end{array}$ & $\begin{array}{l}0.41 \pm \\
0.08 \text { cde }\end{array}$ & $\begin{array}{l}0.57 \pm \\
0.09 \text { ef }\end{array}$ & $\begin{array}{l}0.43 \pm \\
0.05 \text { de }\end{array}$ & $\begin{array}{c}0.38 \pm \\
0.06 \text { bcde }\end{array}$ & $\begin{array}{l}0.59 \pm \\
0.03 \text { ef }\end{array}$ & $\begin{array}{l}0.10 \pm \\
0.03 \mathrm{ab}\end{array}$ & $\begin{array}{l}0.10 \pm \\
0.04 \mathrm{a}\end{array}$ & $\begin{array}{l}0.10 \pm \\
0.02 \mathrm{ab}\end{array}$ & $\begin{array}{l}0.10 \pm \\
0.01 \mathrm{ab}\end{array}$ & $\begin{array}{l}0.010 \pm \\
0.02^{\mathrm{a}}\end{array}$ & $\begin{array}{l}0.11 \pm \\
0.03 \mathrm{abc}\end{array}$ & $\begin{array}{c}\operatorname{tr} \pm \\
0.04 \mathrm{a}\end{array}$ & $\begin{array}{c}0.14 \pm \\
0.09 \mathrm{abcd}\end{array}$ & $* * *$ & $* * *+$ & $* * *$ \\
\hline м23 & $\beta$-cyclocitral & 1230 & A & $\mathrm{nd}^{\mathrm{a}}$ & nd $^{\text {a }}$ & $\mathrm{nd}^{\mathrm{a}}$ & $\mathrm{nd}^{\mathrm{a}}$ & $\mathrm{nd}^{\mathrm{a}}$ & $\mathrm{nd}^{\mathrm{a}}$ & $\mathrm{nd}^{\mathrm{a}}$ & $\mathrm{nd}^{\mathrm{a}}$ & $\begin{array}{l}0.010 \pm \\
0.04 \mathrm{~b}\end{array}$ & $\begin{array}{l}0.12 \pm \\
0.02^{b}\end{array}$ & 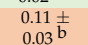 & $\begin{array}{l}0.018 \pm \\
0.02^{b}\end{array}$ & $\begin{array}{l}0.15 \frac{1}{5} \\
0.01 \mathrm{~b}\end{array}$ & $\begin{array}{l}0.12 \pm \\
0.02 \mathrm{~b}\end{array}$ & $\begin{array}{l}0.10 \pm \\
0.01 \mathrm{~b}\end{array}$ & $0.14 \mathrm{t}$ & $* * *$ & $x * *$ & $* * *$ \\
\hline M24 & L-carvone & 1248 & A & $\begin{array}{c}0.96 \pm \\
0.19 \mathrm{bcd}\end{array}$ & $\begin{array}{l}0.57 \pm \\
0.11 \mathrm{abc}\end{array}$ & $\begin{array}{l}1.5 \pm \\
0.05 \mathrm{~d}\end{array}$ & $\begin{array}{l}0.71 \pm \overline{1 \pm} \\
0.06 \mathrm{abc}\end{array}$ & $\begin{array}{c}0.81 \pm \\
0.13 \text { abcd }\end{array}$ & $\begin{array}{l}0.61 \pm \\
0.14 \mathrm{abc}\end{array}$ & $\begin{array}{r}0.75 \pm \\
0.17 \text { abcd }\end{array}$ & $\begin{array}{l}1.1 \pm \pm \\
0.12 \mathrm{~cd}\end{array}$ & $\begin{array}{l}0.38 \\
0.22 \\
0.22 \\
\mathrm{abc}\end{array}$ & $\begin{array}{l}0.26 \pm \\
0.11 \mathrm{ab}\end{array}$ & $\begin{array}{l}0.06 \mathrm{ab} \\
0.038\end{array}$ & $\begin{array}{l}0.02{ }^{0.2 \pm} \\
0.02^{\mathrm{a}}\end{array}$ & $\begin{array}{l}0.010 \\
0.23 \pm \\
0.08\end{array}$ & $\begin{array}{l}0.02 \\
0.36 \pm \\
0.03 \mathrm{abc}\end{array}$ & $\begin{array}{l}0.01 \mathrm{v} \\
0.17 \\
0.0 \mathrm{ab}\end{array}$ & $\begin{array}{l}0.06 \mathrm{0} \\
0.45 \mathrm{abc} \\
0.02 \mathrm{abc}\end{array}$ & $* * *$ & $* * *$ & $* * *$ \\
\hline M25 & D-carvone & 1262 & A & $\begin{array}{c}0.43 \pm \\
0.19\end{array}$ & $\begin{array}{c}0.36 \pm \\
0.10\end{array}$ & $\begin{array}{l}0.24 \pm \\
0.02\end{array}$ & $\begin{array}{c}0.18 \pm \\
0.03\end{array}$ & $\begin{array}{c}0.23 \pm \\
0.08\end{array}$ & $\begin{array}{c}0.34 \pm \\
0.15\end{array}$ & $\begin{array}{c}0.144 \pm \\
0.07\end{array}$ & $\begin{array}{l}0.29 \pm \\
0.06\end{array}$ & $\begin{array}{l}0.33 \pm \\
0.13\end{array}$ & $\begin{array}{l}0.27 \pm \\
0.06\end{array}$ & $\begin{array}{l}0.00 \pm \\
0.13\end{array}$ & $\begin{array}{l}0.36 \pm \\
0.17 \\
0.17\end{array}$ & $\begin{array}{l}0.00 \pm \\
0.30 \pm \\
0.10\end{array}$ & $0.48 \pm$ & $\begin{array}{l}0.00 \pm \\
0.52 \pm \\
0.11\end{array}$ & $\begin{array}{l}0.47 \pm \\
0.18\end{array}$ & ns & ns & ns \\
\hline M26 & thymol & 1290 & A & $\begin{array}{l}0.19 \pm \\
0.05^{\mathrm{b}}\end{array}$ & $\begin{array}{l}0.11 \\
0.14 \mathrm{ab}\end{array}$ & $\begin{array}{l}0.02 \\
0.04 \\
0.04 \mathrm{ab}\end{array}$ & $\begin{array}{l}0.15 \\
0.09 \mathrm{ab} \\
0.09 \mathrm{~b}\end{array}$ & $\begin{array}{l}0.11 \\
0.08 \mathrm{ab} \\
0.06\end{array}$ & $\begin{array}{l}0.10 \\
0.10 \pm \\
0.03 \mathrm{ab}\end{array}$ & $\mathrm{nd}^{\mathrm{a}}$ & $\begin{array}{l}0.14 \\
0.14 \\
0.11 \mathrm{ab}\end{array}$ & $\begin{array}{l}0.13 \\
0.15 \pm \\
0.09 a b\end{array}$ & $\begin{array}{l}0.12 \\
0.12 \mathrm{ab} \\
0.07 \mathrm{ab}\end{array}$ & $\begin{array}{l}0.13 \\
0.15 \\
0.01 \mathrm{ab}\end{array}$ & $\begin{array}{l}0.17 \\
0.16 \pm \\
0.1 \mathrm{ab}\end{array}$ & $\begin{array}{l}0.10 \\
0.12 \pm \\
0.01\end{array}$ & $\begin{array}{l}0.11 \\
0.19 \pm \\
0.00^{\mathrm{b}}\end{array}$ & $\begin{array}{l}0.111 \\
0.10 \pm \\
0.30 \mathrm{ab}\end{array}$ & $\begin{array}{l}0.18 \\
0.16+ \\
0.05 a b\end{array}$ & * & $*$ & * \\
\hline \multirow[t]{2}{*}{ M27 } & $\begin{array}{c}\text { carvacrol } \\
\text { Total }\end{array}$ & 1317 & A & $\begin{array}{l}0.05 \\
0.54 \pm \\
0.08 \\
61\end{array}$ & $\begin{array}{c}0.42 \pm \\
0.09 \\
64\end{array}$ & $\begin{array}{c}0.045 \pm \\
0.045 \\
00 \\
50\end{array}$ & $\begin{array}{c}0.090 \pm \\
0.60 \pm \\
0.02 \\
56\end{array}$ & $\begin{array}{c}0.29 \pm \\
0.03 \\
59\end{array}$ & $\begin{array}{l}0.039 \pm \\
0.03 \\
53 \\
53\end{array}$ & $\begin{array}{c}0.18 \pm \\
0.04 \\
42\end{array}$ & $\begin{array}{c}0.52 \pm \\
0.04 \\
54\end{array}$ & $\begin{array}{c}0.09 \\
0.44 \pm \\
0.21 \\
27\end{array}$ & $\begin{array}{c}0.36 \pm \\
0.27 \\
34\end{array}$ & $\begin{array}{c}0.45 \pm \\
0.05^{\mathrm{a}} \\
42\end{array}$ & $\begin{array}{c}0.53 \pm \\
0.08 \\
38\end{array}$ & $\begin{array}{l}0.011 \pm \\
0.311 \\
0.12 \\
26\end{array}$ & $\begin{array}{c}0.008 \pm \\
0.23 \\
0.23 \\
27\end{array}$ & $\begin{array}{c}0.19 \pm \\
0.07 \\
29\end{array}$ & $\begin{array}{c}0.039 \pm \\
0.39 \\
0.14 \\
30\end{array}$ & ns & ns & ns \\
\hline & $\begin{array}{l}\text { Monoterpenoid } \\
\text { Alcoohols }\end{array}$ & & & & & & & & & & & & & & & & & & & & & \\
\hline MA1 & $\begin{array}{l}\text { (+)-(E)-p-mentha-2,8- } \\
\text { dien-1-ol }\end{array}$ & 1122 & A & $\begin{array}{c}0.10 \pm \\
0.03\end{array}$ & $\begin{array}{c}0.15 \pm \\
0.01\end{array}$ & $\begin{array}{l}\operatorname{tr} \pm \\
0.03\end{array}$ & $\begin{array}{l}0.28 \pm \\
0.03\end{array}$ & $\begin{array}{c}0.10 \pm \\
0.02\end{array}$ & $\begin{array}{c}0.10 \pm \\
0.03\end{array}$ & $\begin{array}{l}\operatorname{tr} \pm \\
0.03\end{array}$ & $\begin{array}{l}0.14 \pm \\
0.01\end{array}$ & $\begin{array}{l}0.15 \pm \\
0.03\end{array}$ & $\begin{array}{c}0.16 \pm \\
0.01\end{array}$ & $\begin{array}{c}0.15 \pm \\
0.03\end{array}$ & $\begin{array}{c}0.13 \pm \\
0.02\end{array}$ & $\begin{array}{l}0.12 \pm \\
0.07\end{array}$ & $\begin{array}{l}0.13 \pm \\
0.02\end{array}$ & $\begin{array}{c}0.12 \pm \\
0.03\end{array}$ & $\begin{array}{l}0.19 \pm \\
0.13\end{array}$ & ns & ns & ns \\
\hline MA2 & dihydrolinalool & 1142 & A & $\mathrm{nd}^{\mathrm{a}}$ & $\mathrm{nd}^{\mathrm{a}}$ & $\mathrm{nd}^{\mathrm{a}}$ & $\mathrm{nd}^{\mathrm{a}}$ & $\mathrm{nd}^{\mathrm{a}}$ & $\mathrm{nd}^{\mathrm{a}}$ & $\mathrm{nd}^{\mathrm{a}}$ & $\mathrm{nd}^{\mathrm{a}}$ & $\begin{array}{l}0.75 \pm \\
0.31 \text { abc }\end{array}$ & $\begin{array}{l}0.33 \\
0.26 \mathrm{abc}\end{array}$ & $\begin{array}{l}0.93 \mathrm{~s} \\
0.08 \mathrm{cc}\end{array}$ & $\begin{array}{l}1.2 \pm \\
0.06^{6}\end{array}$ & $\begin{array}{l}0.78 \\
0.18 \mathrm{abc} \\
0.18\end{array}$ & $\begin{array}{l}0.64 \\
0.64 \\
030 \mathrm{abc}\end{array}$ & $\begin{array}{l}0.29 \\
0.29 \\
0.11 b^{\mathrm{ab}}\end{array}$ & $\begin{array}{l}0.48 \pm \\
0.48 \text { abc }\end{array}$ & $* * *$ & $* * *$ & $* * *$ \\
\hline MA3 & (Z)-pinocarveol & 1147 & В [28] & $\begin{array}{l}0.59 \pm \\
0.13^{\mathrm{a}}\end{array}$ & $\begin{array}{l}0.63 \pm \\
0.17 \mathrm{a}\end{array}$ & $\begin{array}{l}0.30 \pm \\
0.08^{\mathrm{a}}\end{array}$ & $\begin{array}{l}0.20 \pm \\
0.08^{\mathrm{a}}\end{array}$ & $\begin{array}{l}0.28 \pm \\
0.02^{\mathrm{a}}\end{array}$ & $\begin{array}{l}0.35 \pm \\
0.21^{\mathrm{a}}\end{array}$ & $\begin{array}{c}\operatorname{tr} \pm \\
0.06^{\mathrm{a}}\end{array}$ & $\begin{array}{l}0.45 \pm \\
0.10^{\mathrm{a}}\end{array}$ & $\begin{array}{l}0.31 \mathrm{a} \\
0.29 \pm \\
0.09 \mathrm{a}\end{array}$ & $\begin{array}{l}0.26 \mathrm{a} \\
0.21 \pm \\
0.10 \mathrm{a}\end{array}$ & $\begin{array}{l}0.06^{\mathrm{a}} \\
0.08 \mathrm{a} \\
0.01\end{array}$ & $\begin{array}{l}0.00^{\circ} \\
0.01^{\mathrm{a}}\end{array}$ & $\begin{array}{l}0.18 \mathrm{a} \\
0.20 \pm \\
0.10 \mathrm{a}\end{array}$ & $\begin{array}{l}0.30 \mathrm{a} \\
0.47 \pm \\
0.32 \mathrm{a}\end{array}$ & $\begin{array}{l}0.11 \mathrm{a} \\
0.15 \pm \\
0.03 \mathrm{a}\end{array}$ & $\begin{array}{l}0.24 \mathrm{x} \\
0.57 \\
0.42 \mathrm{a}\end{array}$ & * & * & * \\
\hline MA4 & terpinen-4-ol & 1184 & A & $\begin{array}{l}0.10 \pm \\
0.01 \text { ab }\end{array}$ & $\mathrm{nd}^{\mathrm{a}}$ & $\begin{array}{l}\mathrm{tr} \pm \\
0.03^{\mathrm{a}}\end{array}$ & $\begin{array}{c}\mathrm{tr} \pm \\
0.03 \mathrm{ab}\end{array}$ & $\begin{array}{l}\mathrm{tr} \pm \\
0.03^{\mathrm{a}}\end{array}$ & $\begin{array}{l}0.10 \mathrm{a} \\
0.07 \mathrm{ab}\end{array}$ & $\mathrm{nd}^{\mathrm{a}}$ & $\begin{array}{l}0.13 \mathrm{a} \\
0.03 \text { ab }\end{array}$ & $\begin{array}{l}0.010 \mathrm{a} \\
0.09 \mathrm{ab}\end{array}$ & $\begin{array}{l}0.15 \pm \\
0.04 \text { ab }\end{array}$ & $\begin{array}{l}0.013 \pm \\
0.03 \mathrm{ab}\end{array}$ & $\begin{array}{l}0.18 \pm \\
0.02^{\mathrm{b}}\end{array}$ & $\begin{array}{l}0.10 \pm \\
0.04 \mathrm{ab}\end{array}$ & $\begin{array}{l}0.15 \\
0.06 \mathrm{ab}\end{array}$ & $\mathrm{nd}^{\mathrm{a}}$ & $\begin{array}{l}0.20 \pm \\
0.04 \\
0.04\end{array}$ & $* * *$ & $* * *$ & $* * *$ \\
\hline MA5 & $\alpha$-terpineol & 1211 & A & nd & nd & nd & nd & nd & nd & nd & nd & $\begin{array}{l}0.10 \pm \\
0.04\end{array}$ & nd & $\begin{array}{l}0.03 \pm \\
0.01\end{array}$ & $\begin{array}{l}0.010 \pm \\
0.01 \\
0.01\end{array}$ & $\begin{array}{l}0.04 \\
\operatorname{tr} \pm \\
0.03 \\
0.03\end{array}$ & $\begin{array}{l}0.00 \pm \\
0.010\end{array}$ & $\begin{array}{l}\mathrm{tr} \pm \\
0.03\end{array}$ & $\begin{array}{l}0.045 \\
0.09 \\
0.09\end{array}$ & ns & ns & ns \\
\hline MA6 & (E)-8-hydroxylinalool & 1349 & В [19] & $\mathrm{nd}^{\mathrm{a}}$ & $\mathrm{nd}^{\mathrm{a}}$ & $\mathrm{nd}^{\mathrm{a}}$ & $\mathrm{nd}^{\mathrm{a}}$ & $\mathrm{nd}^{\mathrm{a}}$ & $\mathrm{nd}^{\mathrm{a}}$ & $\mathrm{nd}^{\mathrm{a}}$ & $\mathrm{nd}^{\mathrm{a}}$ & $\begin{array}{l}0.19 \\
0.19 \\
0.05\end{array}$ & $\begin{array}{l}0.15 \pm \\
0.06^{\mathrm{b}}\end{array}$ & $\begin{array}{l}0.01 \\
0.00 \pm \\
0.04\end{array}$ & $\begin{array}{l}0.01 \\
0.010 \pm \\
0.01 \mathrm{ab}\end{array}$ & $\begin{array}{l}0.10 \mathrm{~J} \\
0.02 \mathrm{ab}\end{array}$ & $\begin{array}{l}0.01 \\
0.18 \pm \\
0.03 \mathrm{~b}\end{array}$ & $\begin{array}{l}0.10 \\
0.05 \\
0.06 \mathrm{ab}\end{array}$ & $\begin{array}{l}0.09 \\
0.18 \pm \\
0.05^{\mathrm{b}}\end{array}$ & $* * *$ & $* * *$ & $* * *$ \\
\hline \multirow[t]{2}{*}{ MA7 } & caryophylladienol II & 1665 & B [19] & nd a & $\mathrm{nd}^{\mathrm{a}}$ & nd $^{a}$ & nd $^{a}$ & $\mathrm{nd}^{\mathrm{a}}$ & $\mathrm{nd}^{\mathrm{a}}$ & $\mathrm{nd}^{\mathrm{a}}$ & nd $^{a}$ & $0.1 \pm 0.05$ & $\mathrm{nd}^{\mathrm{a}}$ & $\begin{array}{l}0.10 \pm \\
0.1 \mathrm{~b}\end{array}$ & $\begin{array}{l}0.10 \pm \\
0.02\end{array}$ & $\begin{array}{l}0.10 \pm \\
0.15\end{array}$ & $0.11 \pm$ & $\begin{array}{l}0.10 \pm \\
00^{b}\end{array}$ & $\begin{array}{l}0.10 \pm \\
0.03\end{array}$ & $* * *$ & $* * *$ & $* *+$ \\
\hline & $\begin{array}{c}\text { Total } \\
\text { Sesquiterpenes }\end{array}$ & & & 0.79 & 0.78 & 0.38 & 0.53 & 0.39 & 0.48 & 0.06 & 0.72 & 1.6 & 1.0 & 1.6 & 1.9 & 1.4 & 1.8 & $\begin{array}{l}0.027 \\
0.77\end{array}$ & $\begin{array}{l}0.75 \\
1.7\end{array}$ & & & \\
\hline s1 & $\alpha$-ylangene & 1384 & B [27] & $\begin{array}{l}0.26 \pm \\
0.11 \mathrm{c}\end{array}$ & $\begin{array}{l}0.24 \pm \\
0.07 \mathrm{c}\end{array}$ & $\begin{array}{l}0.17 \pm \\
0.11 \mathrm{c}\end{array}$ & $\begin{array}{c}\mathrm{tr} \pm \\
0.01 \mathrm{ab}\end{array}$ & $\begin{array}{l}0.16 \pm \\
0.5 \mathrm{bc}\end{array}$ & $\begin{array}{l}0.19 \pm \\
010^{c}\end{array}$ & $\begin{array}{l}0.22 \pm \\
0.26^{c}\end{array}$ & $\begin{array}{l}0.20 \pm \\
0.14 \mathrm{c}\end{array}$ & $\mathrm{nd}^{\mathrm{a}}$ & $\mathrm{nd}^{\mathrm{a}}$ & $\mathrm{nd}^{\mathrm{a}}$ & $\mathrm{nd}^{\mathrm{a}}$ & $\mathrm{nd}^{\mathrm{a}}$ & $\mathrm{nd}^{\mathrm{a}}$ & $\mathrm{nd}^{\mathrm{a}}$ & $\mathrm{nd}^{\mathrm{a}}$ & $* * *$ & $* * *$ & $* * *$ \\
\hline s2 & $\alpha$-copaene & 1390 & A & $\begin{array}{l}1.1 \pm \\
0.02^{\mathrm{e}}\end{array}$ & $\begin{array}{l}0.06 \pm \\
0.01 \mathrm{de}\end{array}$ & $\begin{array}{l}0.11 \pm \\
0.02 \text { cde } \\
0.03\end{array}$ & $\begin{array}{l}0.10 \pm \\
0.02 \mathrm{ab}\end{array}$ & $\begin{array}{l}0.15 \pm \\
0.05 \mathrm{ab}\end{array}$ & $\begin{array}{l}0.49 \pm \\
0.03 \text { bcd }\end{array}$ & $\begin{array}{l}0.78 \pm \\
0.04 \text { de }\end{array}$ & $\begin{array}{l}0.14 \\
0.77 \\
0.05 \text { de }\end{array}$ & $\begin{array}{l}0.14 \pm \\
0.04 \mathrm{ab}\end{array}$ & $\begin{array}{l}0.09 \pm \\
0.06 \mathrm{ab}\end{array}$ & $\begin{array}{l}0.06 \pm \\
0.02 \mathrm{ab}\end{array}$ & $\mathrm{nd}^{\mathrm{a}}$ & nd $^{a}$ & $\begin{array}{l}0.12 \pm \\
0.05 \mathrm{ab}\end{array}$ & $\begin{array}{l}0.24 \pm \\
0.07 \mathrm{abc}\end{array}$ & $\begin{array}{l}0.22 \pm \\
0.18 \text { abc }\end{array}$ & $* * *$ & $* * *$ & $* * *$ \\
\hline s3 & (E)- $\beta$-caryophyllene & 1430 & В [29] & $\begin{array}{l}\mathrm{t} \pm \\
0.03\end{array}$ & $\begin{array}{l}\operatorname{tr} \pm \\
0.02\end{array}$ & nd & nd & $\begin{array}{l}\mathrm{tr} \pm \\
0.04\end{array}$ & nd & nd & nd & nd & nd & nd & nd & nd & nd & nd & nd & ns & ns & ns \\
\hline 54 & $\beta$-caryophyllene & 1445 & A & $\begin{array}{l}0.03 \\
4.4 \pm \\
0.61\end{array}$ & $\begin{array}{l}0.02 \\
5.5 \pm \\
0.32^{\mathrm{c}}\end{array}$ & $\begin{array}{l}4.1 \pm \pm \\
0.43 \mathrm{bc}\end{array}$ & $\begin{array}{l}2.5 \pm \\
0.39 \mathrm{ab}\end{array}$ & $\begin{array}{l}0.04 \\
4.3 \pm \\
1.3 \mathrm{bc}\end{array}$ & $\begin{array}{l}4.1 \pm \\
1.2 \mathrm{bc}\end{array}$ & $\begin{array}{l}2.4 \pm \\
0.29 \mathrm{ab}\end{array}$ & $\begin{array}{r}2.2 \pm \\
0.50 \mathrm{ab}\end{array}$ & $\begin{array}{l}0.67 \pm \\
0.52^{\mathrm{a}}\end{array}$ & $\begin{array}{l}0.60 \pm \\
0.40^{\mathrm{a}}\end{array}$ & $\begin{array}{l}1.4 \pm \\
0.73^{\mathrm{a}}\end{array}$ & $\begin{array}{l}1.0 \pm \\
0.15^{\mathrm{a}}\end{array}$ & $\begin{array}{l}0.46 \pm \\
0.17^{\mathrm{a}}\end{array}$ & $\begin{array}{l}1.2 \pm \\
0.13^{\mathrm{a}}\end{array}$ & $\begin{array}{l}0.55 \pm \\
0.28 \mathrm{a}\end{array}$ & $\begin{array}{l}0.69 \pm \\
0.28^{\mathrm{a}}\end{array}$ & $* * *$ & $* * *$ & $* * *$ \\
\hline S5 & (+)-aromadendrene & 1452 & A & $\begin{array}{l}0.17 \pm \\
0.04 \mathrm{de}\end{array}$ & $0.21 \pm$ & $\begin{array}{l}0.15 \pm \\
0.04 \text { cde }\end{array}$ & $\begin{array}{l}\mathrm{tr}= \\
0.07 \mathrm{abc}\end{array}$ & $\begin{array}{l}0.13 \pm \\
0.03 \text { cde }\end{array}$ & $\begin{array}{l}0.15 \pm \\
0.08 \text { cde }\end{array}$ & $\begin{array}{l}0.10 \pm \\
0.06 \text { abc }\end{array}$ & $\begin{array}{l}0.10 \pm \\
0.01^{\mathrm{bcd}}\end{array}$ & $\begin{array}{l}\mathrm{tr} \pm \\
0.01 \mathrm{ab}\end{array}$ & $\mathrm{nd}^{\mathrm{a}}$ & $\mathrm{nd}^{\mathrm{a}}$ & $\mathrm{nd}^{\mathrm{a}}$ & $\mathrm{nd}^{\mathrm{a}}$ & $\mathrm{nd}^{\mathrm{a}}$ & $\mathrm{nd}^{\mathrm{a}}$ & $\mathrm{nd}^{\mathrm{a}}$ & $* * *$ & $* * *$ & $* * *$ \\
\hline
\end{tabular}


Table 1. Cont.

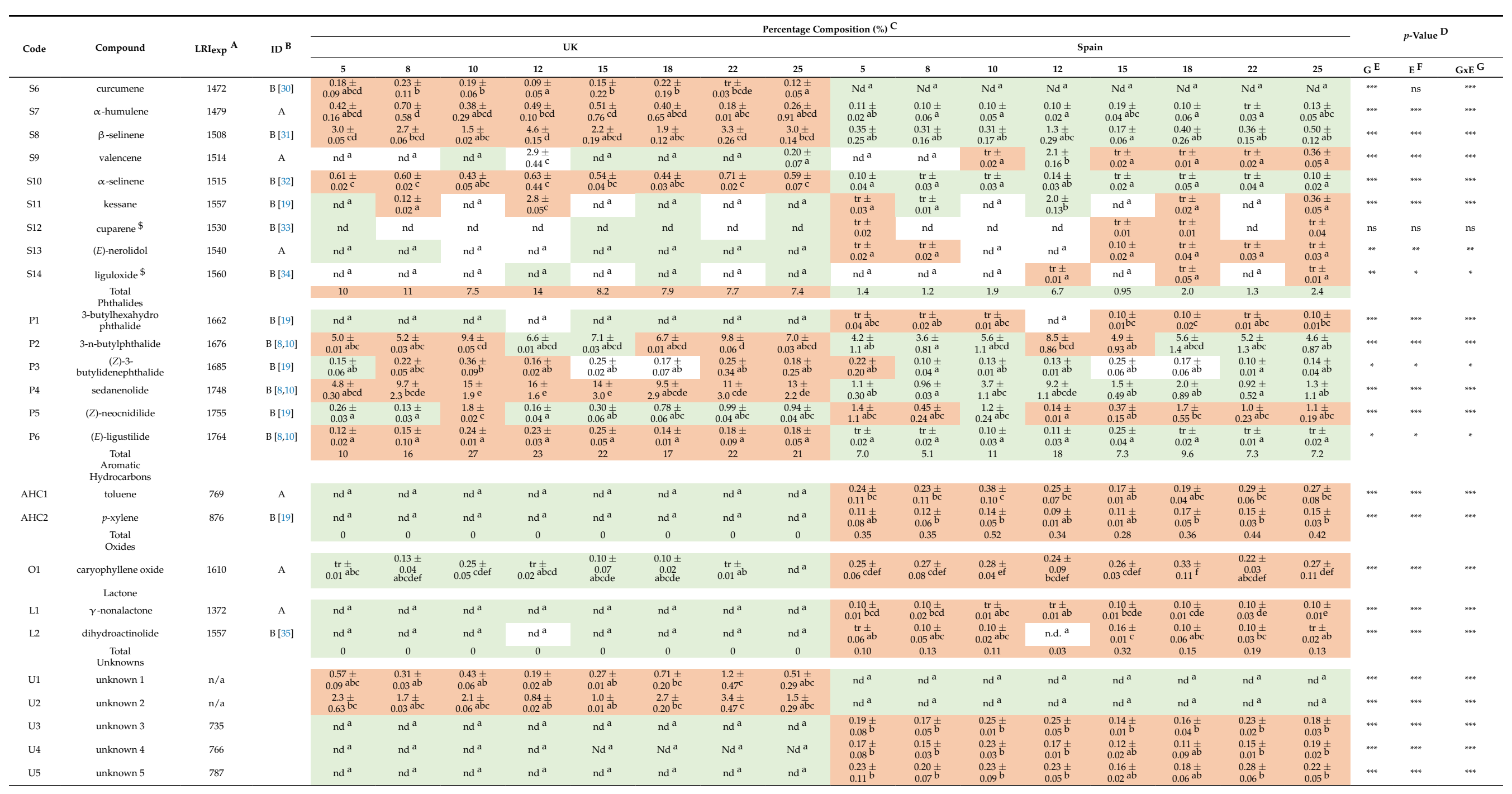


Table 1. Cont.

\begin{tabular}{|c|c|c|c|c|c|c|c|c|c|c|c|c|c|c|c|c|c|c|c|c|c|c|}
\hline \multirow{3}{*}{ Code } & \multirow{3}{*}{ Compound } & \multirow{3}{*}{ LRI $_{\exp }{ }^{A}$} & \multirow{3}{*}{$\mathrm{ID}^{\mathrm{B}}$} & \multicolumn{16}{|c|}{ Percentage Composition (\%) C } & \multirow{2}{*}{\multicolumn{3}{|c|}{$p$-value $\mathrm{D}$}} \\
\hline & & & & \multicolumn{8}{|c|}{ UK } & \multicolumn{8}{|c|}{ Spain } & & & \\
\hline & & & & 5 & 8 & 10 & 12 & 15 & 18 & 22 & 25 & 5 & 8 & 10 & 12 & 15 & 18 & 22 & 25 & $\mathrm{G}^{\mathrm{E}}$ & ${ }_{E} F$ & GXE G \\
\hline U6 & unknown 6 & 896 & & $\mathrm{nd}^{\mathrm{a}}$ & $\mathrm{nd}^{\mathrm{a}}$ & nd $^{\text {a }}$ & $\mathrm{nd}^{\mathrm{a}}$ & $\mathrm{nd}^{\mathrm{a}}$ & $\mathrm{nd}^{\mathrm{a}}$ & $\mathrm{nd}^{\mathrm{a}}$ & $\mathrm{nd}^{\mathrm{a}}$ & $\begin{array}{l}0.22 \pm \\
0.09 \mathrm{~b}\end{array}$ & $\begin{array}{l}0.16 \pm \\
0.04 \mathrm{~b}\end{array}$ & $\begin{array}{l}0.25 \mathrm{t} \\
0.07^{\mathrm{b}}\end{array}$ & $\begin{array}{l}0.22 \pm \\
0.05\end{array}$ & $\begin{array}{l}0.17 \pm \\
0.01 \mathrm{~b}\end{array}$ & $\begin{array}{l}0.22 \mathrm{t} \\
0.03 \mathrm{~b}\end{array}$ & $\begin{array}{l}0.22 \mathrm{t} \\
0.05\end{array}$ & $\begin{array}{l}0.16 \frac{\mathrm{t}}{0.06^{\mathrm{b}}} \\
0\end{array}$ & $* * *$ & $* * *$ & $* * *$ \\
\hline U7 & unknown 7 & 971 & & nd $^{\text {a }}$ & $\mathrm{nd}^{\mathrm{a}}$ & $\mathrm{nd}^{\mathrm{a}}$ & $\mathrm{nd}^{\mathrm{a}}$ & $\mathrm{nd}^{\mathrm{a}}$ & $\mathrm{nd}^{\mathrm{a}}$ & $\mathrm{nd}^{\mathrm{a}}$ & $\mathrm{nd}^{\mathrm{a}}$ & $\begin{array}{l}0.64 \pm \\
0.044^{\mathrm{bc}}\end{array}$ & $\begin{array}{l}0.52 \mathrm{a} \\
0.06 \mathrm{ab}\end{array}$ & $\begin{array}{l}1.1 \pm \\
0.01 \mathrm{c}\end{array}$ & $\begin{array}{l}0.78 \pm \\
0.17 \mathrm{bc}\end{array}$ & $\begin{array}{l}0.42 \pm \\
0.04 \mathrm{ab}\end{array}$ & $\begin{array}{l}0.58 \pm \\
0.02 \mathrm{bc}\end{array}$ & $\begin{array}{l}0.64 \pm \\
0.05 \mathrm{bc}\end{array}$ & $\begin{array}{l}0.73 \pm \\
0.03 \mathrm{~b}\end{array}$ & $* * *$ & $* * *$ & $* * *$ \\
\hline U8 & unknown 8 & 1249 & & $\mathrm{nd}^{\mathrm{a}}$ & $\mathrm{nd}^{\mathrm{a}}$ & $\mathrm{nd}^{\mathrm{a}}$ & $\mathrm{nd}^{\mathrm{a}}$ & $\mathrm{nd}^{\mathrm{a}}$ & $\mathrm{nd}^{\mathrm{a}}$ & $\mathrm{nd}^{\mathrm{a}}$ & $\mathrm{nd}^{\mathrm{a}}$ & $\begin{array}{l}0.54 \pm \\
0.18^{\mathrm{b}}\end{array}$ & $\begin{array}{l}0.06 \mathrm{G} \\
0.06^{\mathrm{b}}\end{array}$ & $\begin{array}{l}0.65 \mathrm{E} \\
0.06^{\mathrm{b}}\end{array}$ & $\begin{array}{l}0.17 \mathrm{t} \\
0.02^{\mathrm{b}}\end{array}$ & $\begin{array}{l}0.04 \mathrm{~s} \\
0.033^{\mathrm{b}}\end{array}$ & $\begin{array}{l}0.56 \mathrm{t} \\
0.13 \mathrm{~b}\end{array}$ & $\begin{array}{l}0.05 \mathrm{t} \\
0.055^{\mathrm{b}}\end{array}$ & $\begin{array}{l}0.03 \\
0.02^{\mathrm{b}}\end{array}$ & $* * *$ & $* * *$ & $* * *$ \\
\hline U9 & unknown 9 & 1279 & & $\begin{array}{l}0.16 \pm \\
0.06 \mathrm{ab}\end{array}$ & $\begin{array}{l}0.08 \pm \\
0.01 \mathrm{a}\end{array}$ & $\begin{array}{l}0.10 \pm \\
0.01 \mathrm{a}\end{array}$ & $\begin{array}{l}0.13 \pm \\
0.03 \mathrm{a}\end{array}$ & $\begin{array}{l}0.24 \pm \pm \\
0.01 \mathrm{ab}\end{array}$ & $\begin{array}{l}0.11 \pm \\
0.01 \mathrm{a}\end{array}$ & $\begin{array}{l}0.17 \pm \pm \\
0.03 \mathrm{ab}\end{array}$ & $\begin{array}{l}0.10 \pm \pm \\
0.04 \mathrm{ab}\end{array}$ & $\begin{array}{l}0.18 \\
0.12 \mathrm{ab} \\
0.12 \mathrm{ab}\end{array}$ & $\begin{array}{l}0.06 \\
0.18 \\
0.06 \text { ab }\end{array}$ & $\begin{array}{l}0.06 \mathrm{c} \\
0.07 \mathrm{ab}\end{array}$ & $\begin{array}{l}0.02 \\
0.02 \text { 列 } \\
0.02 \mathrm{~b}\end{array}$ & $\begin{array}{l}0.05 \\
0.05 \\
0.05 \mathrm{ab}\end{array}$ & $\begin{array}{l}0.13 \\
0.22 \pm \\
0.05 \mathrm{ab}\end{array}$ & $\begin{array}{l}0.05 \\
0.04 \pm \\
0.04\end{array}$ & $\begin{array}{l}0.020 \pm \\
0.50 \pm \\
0.19 \mathrm{bc}\end{array}$ & * & * & * \\
\hline U10 & unknown 10 & 1362 & & $\begin{array}{l}0.10 \pm \\
0.02 \mathrm{ab}\end{array}$ & $\begin{array}{l}0.09 \\
0.03 \mathrm{ab}\end{array}$ & $\mathrm{nd}^{\mathrm{a}}$ & $\begin{array}{l}0.16 \mathrm{~s} \\
0.01 \mathrm{~b}\end{array}$ & $\begin{array}{l}0.03 \pm \\
0.044^{\mathrm{a}}\end{array}$ & $\begin{array}{l}0.110 \pm \\
0.01 \mathrm{ab}\end{array}$ & $\begin{array}{l}0.08 \mathrm{a} \\
0.01 \mathrm{ab}\end{array}$ & $\begin{array}{l}0.07 \pm \\
0.4^{\mathrm{a}}\end{array}$ & $\mathrm{nd}^{\mathrm{a}}$ & nd $^{a}$ & $\mathrm{nd}^{\mathrm{a}}$ & nd $\mathrm{a}^{\mathrm{a}}$ & nd ${ }^{\text {a }}$ & $\mathrm{nd}^{\mathrm{a}}$ & nd ${ }^{\text {a }}$ & $\mathrm{nd}^{\mathrm{a}}$ & $* * *$ & $* *$ & $* * *$ \\
\hline U11 & unknown 11 & 1506 & & $\mathrm{nd}^{\mathrm{a}}$ & nd $\mathrm{a}^{\mathrm{a}}$ & $\mathrm{nd}^{\mathrm{a}}$ & $\mathrm{nd}^{\mathrm{a}}$ & $n^{a} d^{a}$ & $\mathrm{nd}^{\mathrm{a}}$ & nd $\mathrm{a}^{\mathrm{a}}$ & $\mathrm{nd}^{\mathrm{a}}$ & $\begin{array}{l}0.10 \pm 0 \\
0.05 \mathrm{ab}\end{array}$ & $\begin{array}{l}0.10 \pm \\
0.01 \mathrm{ab}\end{array}$ & $\begin{array}{l}0.13 \pm \\
0.04^{\mathrm{b}}\end{array}$ & $\begin{array}{l}0.10 \pm \\
0.05 \text { ab }\end{array}$ & $\begin{array}{l}0.10 \pm \\
0.03^{\mathrm{a}}\end{array}$ & $\begin{array}{l}0.13 \pm \\
0.05 \mathrm{~b}\end{array}$ & $\begin{array}{l}0.13 \pm \\
0.03 \mathrm{~b}\end{array}$ & $\begin{array}{l}0.13 \pm \\
0.06 \mathrm{~b}\end{array}$ & $* *$ & $* * *$ & $* * *$ \\
\hline U12 & unknown 12 & 1539 & & $\begin{array}{l}0.25 \pm \\
0.202 \mathrm{ab}\end{array}$ & $\begin{array}{l}0.33 \pm \\
0.04 \mathrm{~b}\end{array}$ & $\begin{array}{l}0.19 \mathrm{a} \\
0.02 \mathrm{ab}\end{array}$ & $\begin{array}{l}0.13 \pm \\
0.01 \mathrm{a}\end{array}$ & $\begin{array}{l}0.10 \pm \pm \\
0.04 \mathrm{ab}\end{array}$ & $\begin{array}{l}0.10 \pm \\
0.01 \mathrm{a}\end{array}$ & $\begin{array}{l}0.18 \mathrm{~A} \\
0.01 \mathrm{ab}\end{array}$ & $\begin{array}{l}0.12 \pm \\
0.04 \mathrm{ab}\end{array}$ & $\begin{array}{l}0.10 \pm \\
0.04 \mathrm{a} \\
0.04\end{array}$ & $\begin{array}{l}0.010 \pm \\
0.077^{\mathrm{a}}\end{array}$ & $\begin{array}{l}0.047 \pm \\
0.04 \mathrm{ab}\end{array}$ & $\begin{array}{l}0.020 \pm \\
0.202 \mathrm{ab}\end{array}$ & $\begin{array}{l}0.11 \pm \\
0.02 \mathrm{a}\end{array}$ & $\begin{array}{l}0.03 \\
0.07 \text { ab } \\
0.07\end{array}$ & $\begin{array}{l}0.030 \pm \\
0.01 \mathrm{a}\end{array}$ & $\begin{array}{l}0.06 \mathrm{c} \\
0.06 \mathrm{ab} \\
0.06\end{array}$ & $* *$ & $* *$ & $* *$ \\
\hline U13 & unknown 13 & 1684 & & $\mathrm{nd}^{\mathrm{a}}$ & $\mathrm{nd}^{\mathrm{a}}$ & $\mathrm{nd}^{\mathrm{a}}$ & $\mathrm{nd}^{\mathrm{a}}$ & $\mathrm{nd}^{\mathrm{a}}$ & $\mathrm{nd}^{\mathrm{a}}$ & $\mathrm{nd}^{\mathrm{a}}$ & $\mathrm{nd}^{\mathrm{a}}$ & $\begin{array}{l}\mathrm{tr} \pm \\
0.06 \mathrm{a}\end{array}$ & $\begin{array}{l}\mathrm{tt} \pm \\
0.02 \mathrm{a}\end{array}$ & $\begin{array}{l}\mathrm{tr} \pm \\
0.02 \mathrm{a}\end{array}$ & $\begin{array}{c}\mathrm{t} \pm \\
0.03 \mathrm{a}\end{array}$ & $\begin{array}{l}\mathrm{tr} \pm \\
0.02 \mathrm{a}\end{array}$ & $\begin{array}{l}0.10 \pm \\
0.01 \mathrm{a}\end{array}$ & $\begin{array}{l}\mathrm{t}+ \pm \\
0.02 \mathrm{a}\end{array}$ & $\begin{array}{l}\text { tr } \pm \\
0.01 \mathrm{a}\end{array}$ & $*$ & $* *$ & $*$ \\
\hline U14 & unknown 14 & 1706 & & $\mathrm{nd}^{\mathrm{a}}$ & $\mathrm{nd}^{\mathrm{a}}$ & $\mathrm{nd}^{\mathrm{a}}$ & $\mathrm{nd}^{\mathrm{a}}$ & $\mathrm{nd}^{\mathrm{a}}$ & $\mathrm{nd}^{\mathrm{a}}$ & $\mathrm{nd}^{\mathrm{a}}$ & $\mathrm{nd}^{\mathrm{a}}$ & $\begin{array}{l}0.100 \\
0.09 \mathrm{ab} \\
0.09\end{array}$ & $\begin{array}{c}\mathrm{tr} \\
\mathrm{t} \pm \mathrm{t} \\
0.02 \mathrm{ab}\end{array}$ & $\begin{array}{l}0.02 \mathrm{c} \\
0.02 \mathrm{ab}\end{array}$ & $\begin{array}{l}0.11 \pm \\
0.01 \mathrm{~b}\end{array}$ & $\begin{array}{l}0.02 \\
0.04= \\
0.04 \mathrm{ab}\end{array}$ & $\begin{array}{l}0.13 \pm \\
0.02^{\mathrm{b}}\end{array}$ & $\begin{array}{l}0.02 \\
0.03= \pm \\
0.03 \mathrm{ab}\end{array}$ & $\begin{array}{l}0.10 \pm \\
0.05 \mathrm{ab}\end{array}$ & $* * *$ & $* * *$ & $* * *$ \\
\hline \multirow[t]{2}{*}{ U15 } & unknown 15 & 1799 & & $\mathrm{nd}^{\mathrm{a}}$ & $\mathrm{nd}^{\mathrm{a}}$ & $\mathrm{nd}^{\mathrm{a}}$ & nd $^{a}$ & $\mathrm{nd}^{\mathrm{a}}$ & $\mathrm{nd}^{\mathrm{a}}$ & $\mathrm{nd}^{\mathrm{a}}$ & $\mathrm{nd}^{\mathrm{a}}$ & $\begin{array}{l}0.13 \pm \\
0.03 \\
0.03\end{array}$ & $\begin{array}{l}0.02 \pm 0.05 \\
0.05 \\
0.5\end{array}$ & $\begin{array}{l}0.02 \\
0.018 \\
0.01\end{array}$ & $\begin{array}{l}0.13 \pm \\
0.04 \mathrm{~b}\end{array}$ & $\begin{array}{l}0.07 \\
0.010 \pm \\
0.01 \mathrm{~b}\end{array}$ & $\begin{array}{l}0.18 \mathrm{z} \\
0.04 \mathrm{~b}\end{array}$ & $\begin{array}{l}0.012 \pm \\
0.02 \mathrm{~b}\end{array}$ & $\begin{array}{l}0.03 \\
0.05^{\circ}\end{array}$ & $* * *$ & $* * *$ & $* * *$ \\
\hline & Total & & & 3.4 & 2.5 & 2.9 & 1.4 & 1.8 & 3.8 & 5.1 & 2.4 & 2.7 & 2.2 & 3.5 & ${ }_{3.0}^{0.04}$ & 2.2 & 2.7 & 2.6 & 3.0 & & & \\
\hline
\end{tabular}

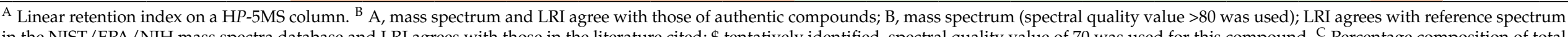

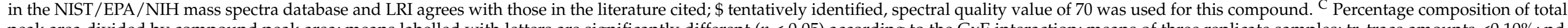

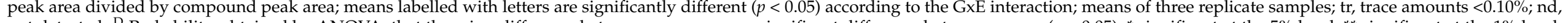

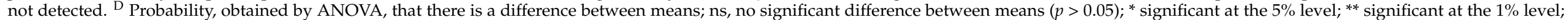

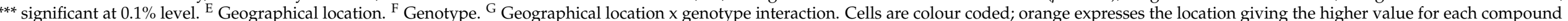
for each genotype; green expresses the location giving the lower value of each compound for each genotype; no colour expresses no difference in percentage composition for both locations. 
As observed in various studies, monoterpenes, sesquiterpenes and phthalides are the most reported compound groups to contribute to celery's aroma profile [4-6,8,36,37]. The composition of celery grown in UK expressed an average of 55\% monoterpenes, $20 \%$ phthalides and $9.2 \%$ sesquiterpenes, whereas genotypes grown in Spain had an average of $32 \%, 2.2 \%$ and $9 \%$, respectively. Monoterpenes comprised most of the composition of the aroma profile of all celery genotypes grown in the UK, with limonene, $\gamma$-terpinene, $\beta$-pinene and $m$-cymene exhibiting the highest proportion of monoterpenes $[4,7]$. A lower proportion of monoterpenes comprised Spanish-grown celery, however, genotypes 10 and 12 displayed over $10 \%$ more than the other genotypes (Table 1 ). The authors previously carried out gas chromatography-olfactometry (GC/O) on two celery genotypes (12 and 25 ) and reported that these compounds contribute citrus, fresh, pine, and mint odours to celery [8]. Although these compounds comprised much of the aroma profile, their odour activity remains low and, therefore, they would not be considered characteristic compounds to celery. By completing aroma extraction dilution analysis (AEDA), Kurobayashi, Kouno, Fujita, Morimitsu and Kubota [38] identified the flavour dilution (FD) factor of volatile compounds of raw and boiled celery. Phthalides including 3-n-butylphthalide and ligustilide were found to have the highest FD factor of 3125 , whereas myrcene, a monoterpene also identified within the current study, had a FD value of 625. Uhlig, Chang and Jen [3] investigated the effect of phthalides on celery flavour using eight celery cultivars of varying origins, observing a positive correlation with total phthalide content and the intensity of the 'celery flavour' attribute. Significant variation between celery cultivars and phthalide content was also observed, most obviously in the concentration of sedanenolide. This is reflected in the current study.

The prominence of phthalides and their contribution to celery aroma is apparent throughout literature. A review completed by the authors [7] identified 3-n-butylphthalide and sedanenolide to be the most reported phthalides in celery, with odour descriptors such as celery, herbal and cooked celery. These compounds have been identified as characteristic compounds to celery aroma, and when authors [8] completed GC/O upon two celery genotypes also used in this study $(12,22)$, the average odour intensity of these compounds was high throughout maturity. Growing celery in the UK in 2018 produced genotypes with a higher phthalide composition, particularly high in 3-n-butylphthalide and sedanenolide, comprising an average percentage of $7.1 \%$ and $11.6 \%$, respectively. The average percentage of these compounds was lower in celery growing in Spain in 2019, with 3-n-butylphthalide and sedanenolide contributing an average of $5.3 \%$ and $2.6 \%$, respectively. However, ( $Z$ )neocnidilide was expressed at a higher composition in Spanish celery, comprising an average of $0.92 \%$ of the aroma profile. Pino, Rosado and Fuentes [39] identified sedanenolide to comprise much of the volatile profile of celery leaf oil, comprising $32.1 \%$ of the composition. The significantly higher abundance of these phthalide compounds, reflected in Table 1, will allow assumptions to be drawn that these genotypes have a stronger typical celery aroma [3].

A similar pattern was observed within sesquiterpenes, whereby celery grown in the UK exhibited a significantly higher proportion of sesquiterpenes compared to Spanish grown celery. $\beta$-Caryophyllene and $\beta$-selinene comprised the highest proportion of the sesquiterpene profile for both geographical locations, and these two are the most reported sesquiterpenes in celery $[7,36,37,40]$. A similar sesquiterpene trend was observed in another study [10] between two harvest years (2018 and 2020) for the same eight genotypes, whereby the sesquiterpene content comprised a higher proportion of the volatile profile of celery grown in 2018, a significantly warmer season than 2020 [10]. Pino, Rosado and Fuentes [39] identified $\beta$-caryophyllene to comprise $13.5 \%$ of the volatile profile of Cuban celery leaf oil, whereas Lund, Wagner and Bryan [41] identified $\beta$-caryophyllene and $\beta$-selinene to comprise an average of $1.5 \%$ and $3.4 \%$, respectively. Lund, Wagner and Bryan [41] also identified $\beta$-selinene to have a celery-like odour. 
Whilst monoterpenes formed much of the composition of UK grown celery, aldehydes were observed to contribute a high proportion in Spanish-grown celery for all genotypes, except genotypes 10 and 12 , comprising an average of $38.5 \%$ of the aroma composition. Hexanal and (E)-2-heptenal were the most abundant compounds in this group for both geographical locations and genotypes, with odour characteristics of fresh, green and fatty. Although not identified in UK grown celery, benzaldehyde and (E)-2-octenal composed a high proportion of the volatile composition, with odour characteristics of almond, cherry, and cucumber, averaging to comprise $2.0 \%$ and $2.7 \%$, respectively. Aldehyde content within celery has not been discussed thoroughly, with only a few studies detecting the compound group. Gold and Wilson [9] identified a range of aldehydes including hexanal, octanal and heptanal, yet Shojaei, Ebrahimi and Salimi [40] only identified benzeneacetaldehyde and nonanal within three ecotypes of wild celery. A large proportion of aldehydes that were identified in the current study were detected, using $\mathrm{GC} / \mathrm{O}$, to be prominent throughout celery maturity [8]. Hexanal was one of the compounds contributing the most to the aldehyde content in celery for all genotypes across both locations, with odour characteristics including fresh, green and apple, as well as being identified throughout celery maturity [8].

Similarly, the ketone content of celery has rarely been discussed and only few studies have reported these compounds $[8,9,40]$. Accompanying the identification of aldehydes, Shojaei, Ebrahimi and Salimi [40] further detected $p$-methyl acetophenone and 2 -undecanone within the three wild celery ecotypes. An explanation for the variation in ketone content between geographical location could involve investigating the formation of phthalides. The metabolic pathway involved in the synthesis of phthalides has yet to be confirmed and, currently, there are multiple suggestions looking into how phthalides are synthesised [7]. Phan, Kim, and Dong [42] identified a method of synthesising phthalides through ketone hydroacylation. Here, the hydroacylation of ketones led to the formation of five-membered lactones, inducing the synthesis of $1(3 \mathrm{H})$-isobenzofuranone, the simplest phthalide structure. From here, various phthalides can be formed according to the substitution at C3 [7,42]. The large variety of ketones identified (Table 1) may be an indication of the potential for the Spanish crop to synthesise phthalides. Many ketones were identified by the authors [8] to be important to celery aroma when using GC/O to measure the change in aroma during celery maturity. The compounds 3-Pentanone, 2-hexanone and 3-octen-2-one were detected at higher intensities in immature celery, displaying the crop's potential to synthesis phthalide compounds, whereas 1-octen-3-one was identified by GC/MS with a relative abundance of 6.7 and $4.7 \mathrm{AU}$, respectively, in post-mature celery.

Principal Component Analysis of Volatile Compounds in UK and Spanish Celery Samples

Principal component analysis allowed for the visual comparison of the volatile composition of the eight celery genotypes grown in UK and Spain (Figure 1) and to examine any correlations occurring between genotype, geographical location and chemical compounds. Using only the significant compounds for geographical location $(G)$, genotype $(E)$ and their interaction $(\mathrm{GxE})$, a clear divide between the compounds associated with each year was observed. Principal component one (F1) and two (F2) explained $72.32 \%$ of the total variation present in the data, and it can be observed that the first axis separated samples from the geographical location (UK and Spain), whereas the second axis separated the various genotypes within a location. Differences between geographical location were apparent, as they separated along the F2 component. 


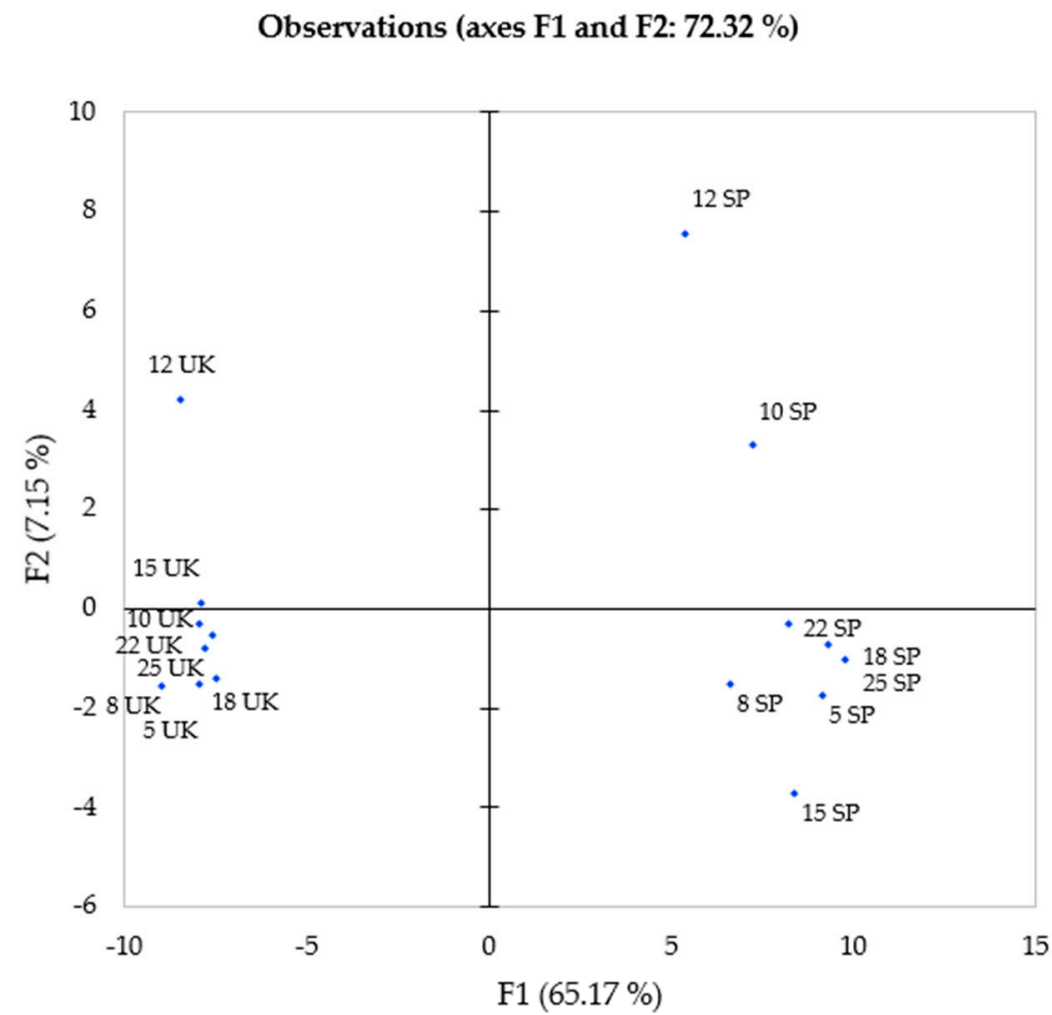

(A)

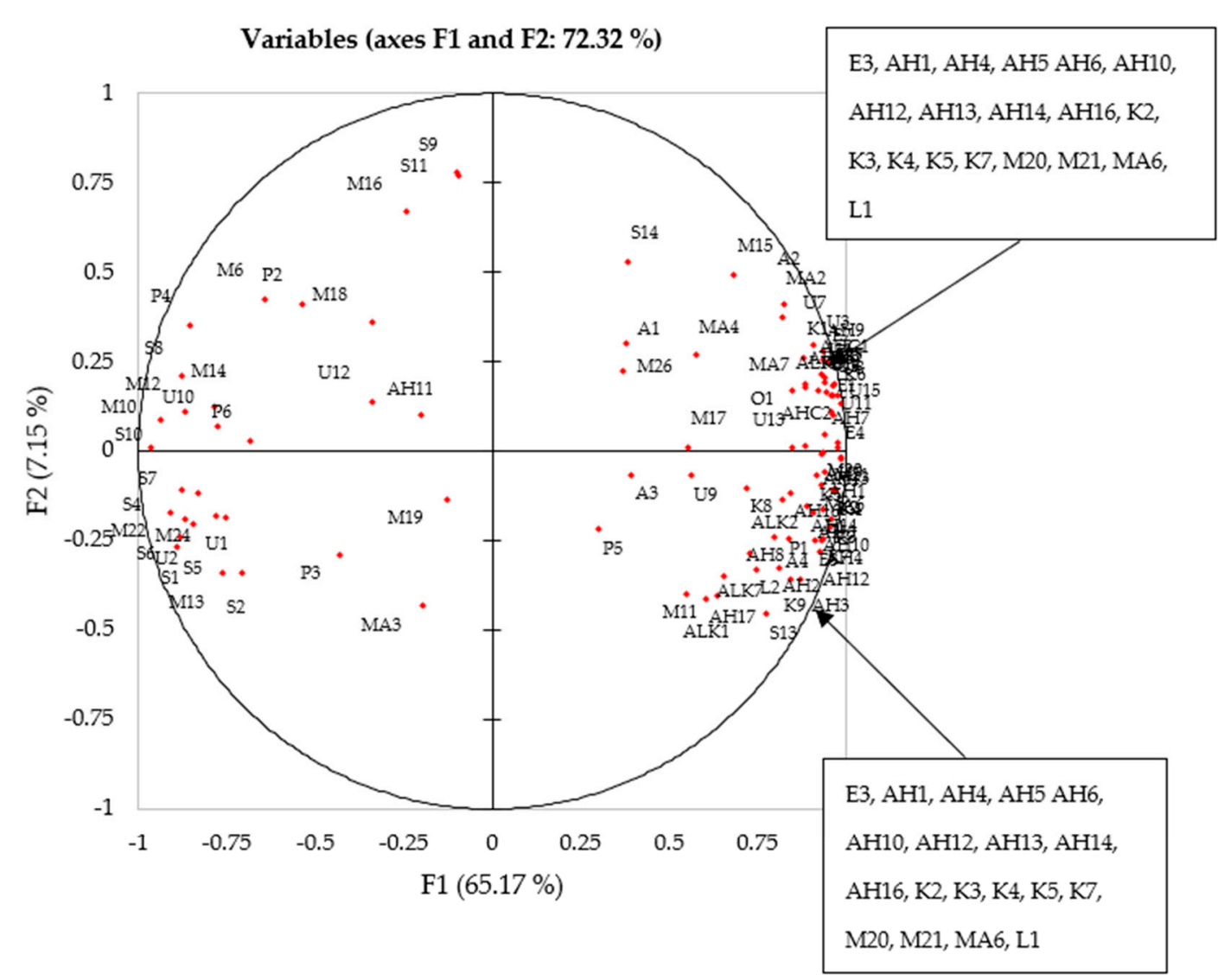

(B)

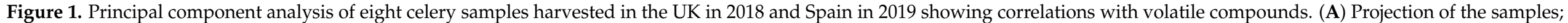
(B) Distribution of variables. 
Genotype expressed a significant influence over both the UK- and Spanish-grown celery (Table 1), yet a more noticeable separation was observed in the Spanish-grown celery between genotypes, in addition to a strong association with more aroma compounds than UK celery (Figure 1). Genotype expressed significant differences (Table 1), but genotypes 12,22 and 25 for Spain were positioned in a similar place on the opposite quadrant in the observation plot. Genotype 12 in both locations took the appearance of an outlier, displayed as the most significantly different from other genotypes used within this experiment. This was caused by the high abundance of sesquiterpene compounds present in the UK harvest, especially from $\beta$-selinene, and the high phthalide content within the Spanish harvest, with 3-n-butylphthalide and sedanenolide comprising $8.5 \%$ and $9.2 \%$, respectively, of the total volatile content. Significant compound associations with Spanish grown celery were expressed within Figure 1 including all aldehydes (except AH11) and ketones, accompanied by monoterpenes (M11, 15, 17, 20, 26), sesquiterpenes $(S 13,14)$, phthalides $(P 1,5)$ and alcohols (A1, 2, 3). This was further reflected in Table 1. Conversely, less noticeable separation between the eight celery genotypes was observed by celery grown in the UK, in addition to fewer compound associations. Monoterpenes (M6, 10, 12, 13, 14, 16, 18, 22, 24), sesquiterpenes $(\mathrm{S} 1,2,4,5,6,7,8,10,12)$ and phthalides $(\mathrm{P} 2,3,4,6)$ were positively correlated with samples grown in the UK. The spread of monoterpenes, sesquiterpenes and phthalides across the plot, together with ubiquity within all celery genotypes regardless of location of growth, harvest year [10] and maturity [8], confirmed the importance of these compound groups to celery and celery aroma. This was originally concluded by the authors [10], where eight genotypes of celery grown in the UK in 2018 and 2020 both exhibited these compounds, and in a similar pattern. Aldehydes and ketones appeared to be more strongly influenced by geographical location rather than genotype, explaining why these compounds are not commonly reported within the celery volatile composition.

Genotype and geographical location both expressed a significant influence over the volatile content of celery (Table 1), however, geographical location expressed a stronger influence upon the composition (Figure 1). Differences within the growing climate and agronomy applied to the celery increased the risk of variation, as similarly expressed between harvest years [10], whereby differences in air temperatures were likely the cause for the large variation expressed between years 2018 and 2020, altering the sensory profile of the crop. The differences in composition observed between the eight celery genotypes grown in the UK and Spain (Figure 1) and the impact that these have upon the sensory characteristics were investigated through sensory profiling.

\subsection{Sensory Evaluation of Fresh Celery Samples}

The sensory profile of the eight celery samples was generated by a trained panel who came to the consensus of 22 and 23 terms for the quantitative assessment of samples grown in the UK in 2018 and samples grown in Spain in 2019, respectively. The additional attribute for the samples grown in Spain in 2019 was salty taste, and we hypothesised that this was because of the saline soils present in this part of the country, as observed in other studies such as tomato [43], pepper [44] and cauliflower [45]. Mean panel scores for these attributes are presented in Table 2. Out of the 22 attributes that were profiled from the UK harvest, 14 of these were found to be significantly different between the genotypes, and seven out of 23 attributes were significantly different for the Spanish trial in 2019. Few significant assessor $x$ sample interactions were identified for both UK and Spanish harvests, suggesting that the panellists scored samples in a consistent manner [46]. Statistical comparison of sensory differences between locations could not be completed due to the one-year difference between harvests, however, general trends will be discussed. 
Table 2. Mean panel scores for sensory attributes of the eight celery samples harvested in UK 2018 and Spain 2019.

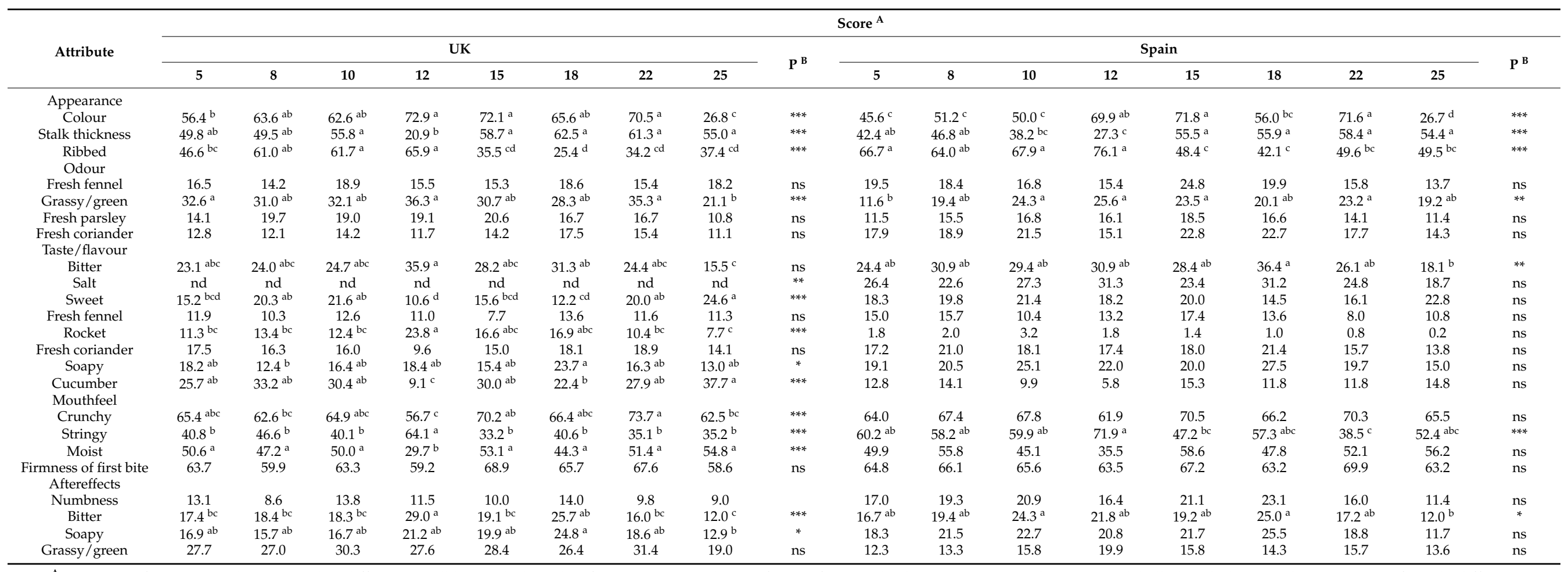

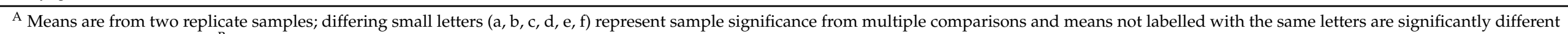

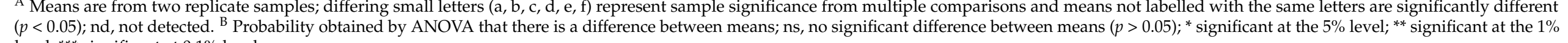
level; *** significant at $0.1 \%$ level. 
Appearance attributes for both locations displayed significant differences caused by genotype, and similarities were observed between scoring for stalk thickness and colour attributes. A significant difference $(p<0.001)$ for ribbed appearance was apparent between locations for all genotypes. The genotype variation between ribbed appearance was more apparent for those harvested in the UK than those harvested in Spain, with scores ranging from 25.4 to 65.9. Mouthfeel attributes displayed a positive correlation with appearance attributes, and these attributes were the highest scoring attributes in all genotypes across both locations, apart from stringiness. Stringiness was scored higher in Spanish celery, with all genotypes of the Spanish celery recording an increase of at least 10, apart from genotype 22. Genotype 22 was scored significantly lower for stringiness when comparing other genotypes in both locations. Although not significantly different, grassy after-effect was scored higher within UK celery and exhibited a positive correlation with grassy odour, an attribute that was significantly different in both locations.Significant differences in the odour and flavour attributes evaluated in both genotypes and geographical location were observed but, more significantly, different attributes were identified in UK celery. The cucumber and rocket flavour with grass odour attributes were scored higher in the UK harvest, whereas Spanish-grown celery scored higher for fresh coriander odour, fennel and soapy flavour. The fresh coriander flavour attribute was scored alike for both locations, however genotype 12 displayed a higher score in coriander flavour when grown in Spain, going from a score of 9.6 to 17.4. Furthermore, genotype 12 was scored as most bitter with genotype 8 and 18 for both locations, but scored sweeter when grown in Spain. Genotype 18 was scored with the strongest soapy flavour, which expressed a positive correlation with fresh fennel. Where genotype 12 scored high for flavour/odour attributes (apart from cucumber), genotype 25 scored low for flavour/odour attributes, only scoring high in the cucumber flavour attribute in both locations.

\section{Principal Component Analysis of Flavour Attributes and Volatile Compounds}

PCA was used to visualise the sensory and chemical differences observed across the eight genotypes, with the volatile compounds identified (Table 1) and the sensory attributes related to odour and flavour used as variables (Figures 2 and 3). Celery grown in the UK expressed a large variation between the eight genotypes (Figure 2), whereby principal component one (F1) and two (F2) explained $69.49 \%$ of the total variation within the data. The first axis separated genotypes 5, 10, 18 and 22 from other genotypes, whereas the second axis separated genotypes 10,12, 15 and 18. Genotype 25 was scored the lowest for all flavour attributes, only scoring high in cucumber flavour (Table 2), whereas genotype 12 opposed genotype 25 (Figure 2) and displayed strong association with a fresh parsley and grass odour along with a rocket flavour. Genotype 18 was positively correlated with fresh fennel and coriander flavour, with the soapy characteristics that accompany many members of the Apiaceae family [47]. A grouping of aroma compounds in the centre of the PCA was observed, whereas the sensory characteristics remained positioned on the outer rim of the biplot, with genotypes 5 and 22 grouped in the middle of the observation plot accompanied with no strong associations with any flavour/odour attribute (Figure 2). These genotypes exhibited a lower volatile content to genotype 12 (Table 1). Predominantly, monoterpenes and sesquiterpenes were negatively correlated with the first principal component (F1), and compounds belonging to compound classes such as alcohols and aldehydes were positively associated with F1. Phthalides were distributed around the plot, with (Z)-neocnidilide (P5) displaying positive association to fresh fennel, whereas sedanenolide and $(E)$-ligustilide (P4 and P6) express a positive correlation with fresh parsley. 


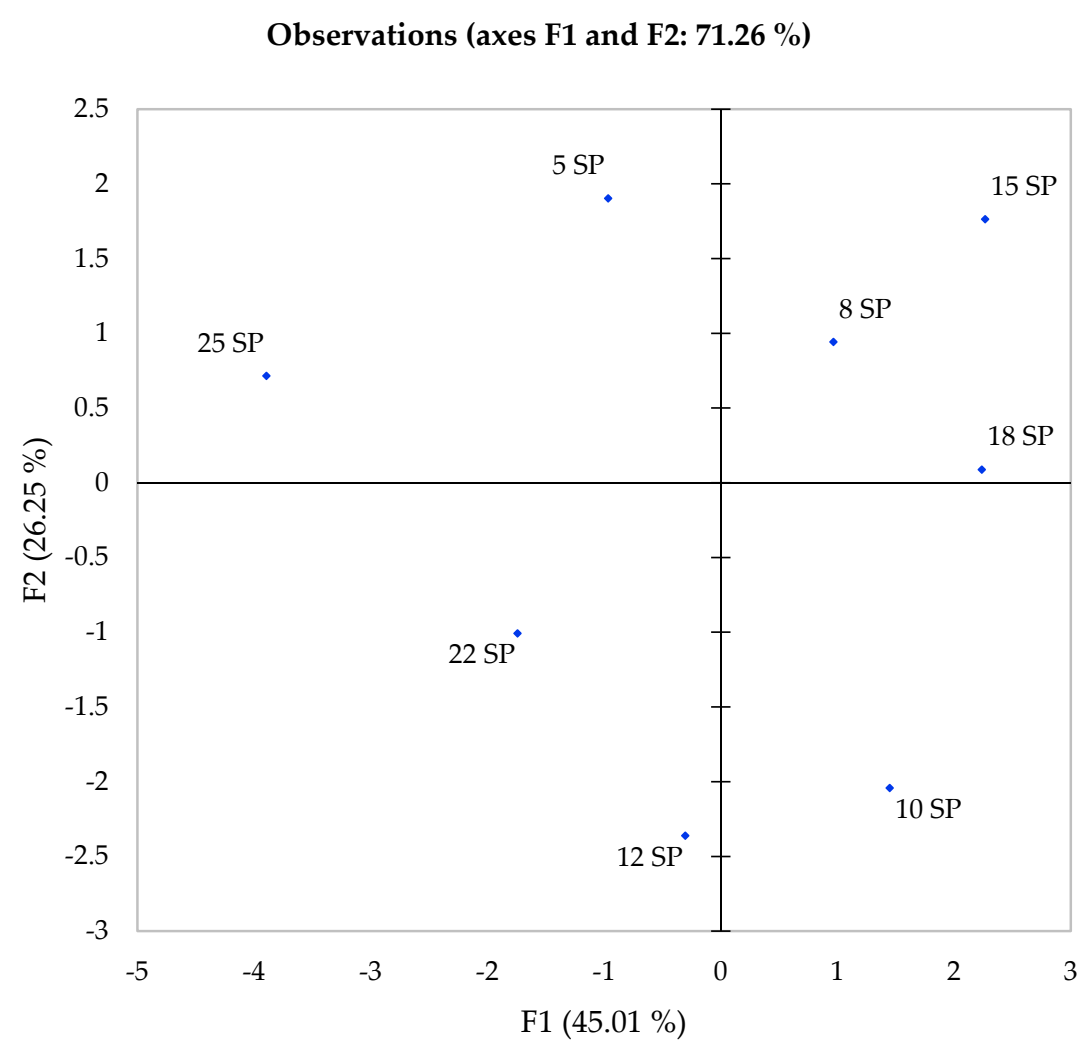

(A)

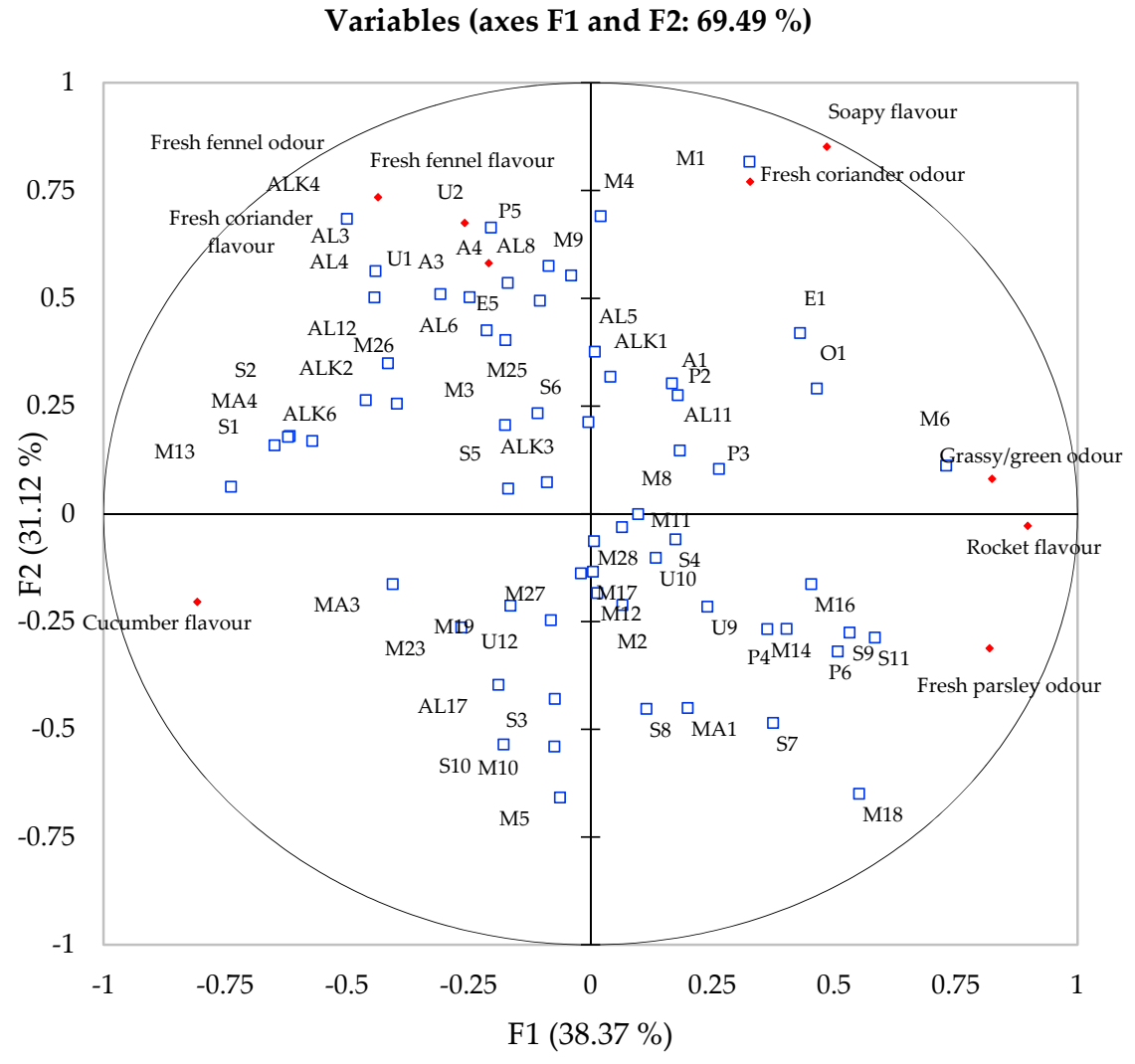

(B)

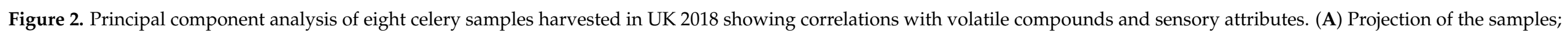
(B) Distribution of variables. 


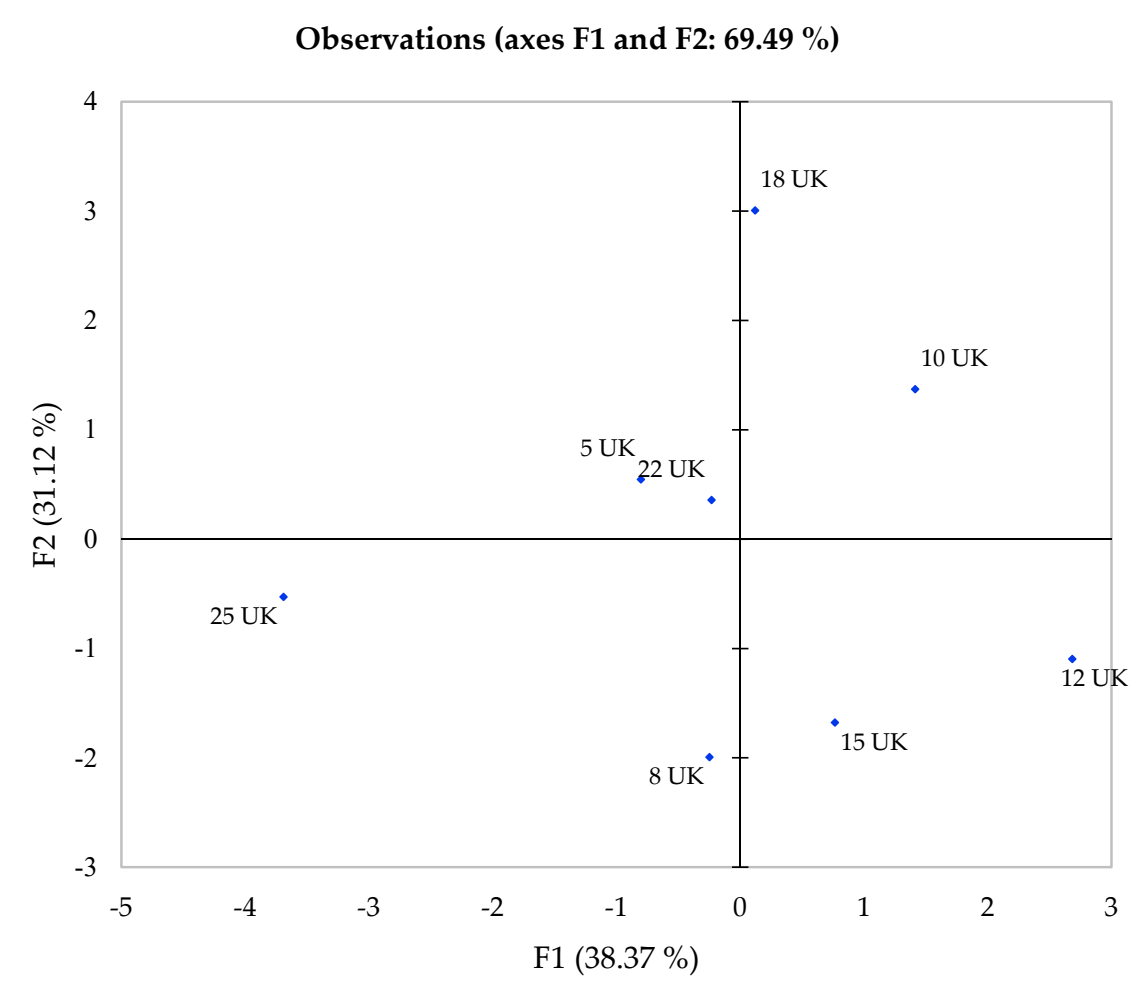

(A)

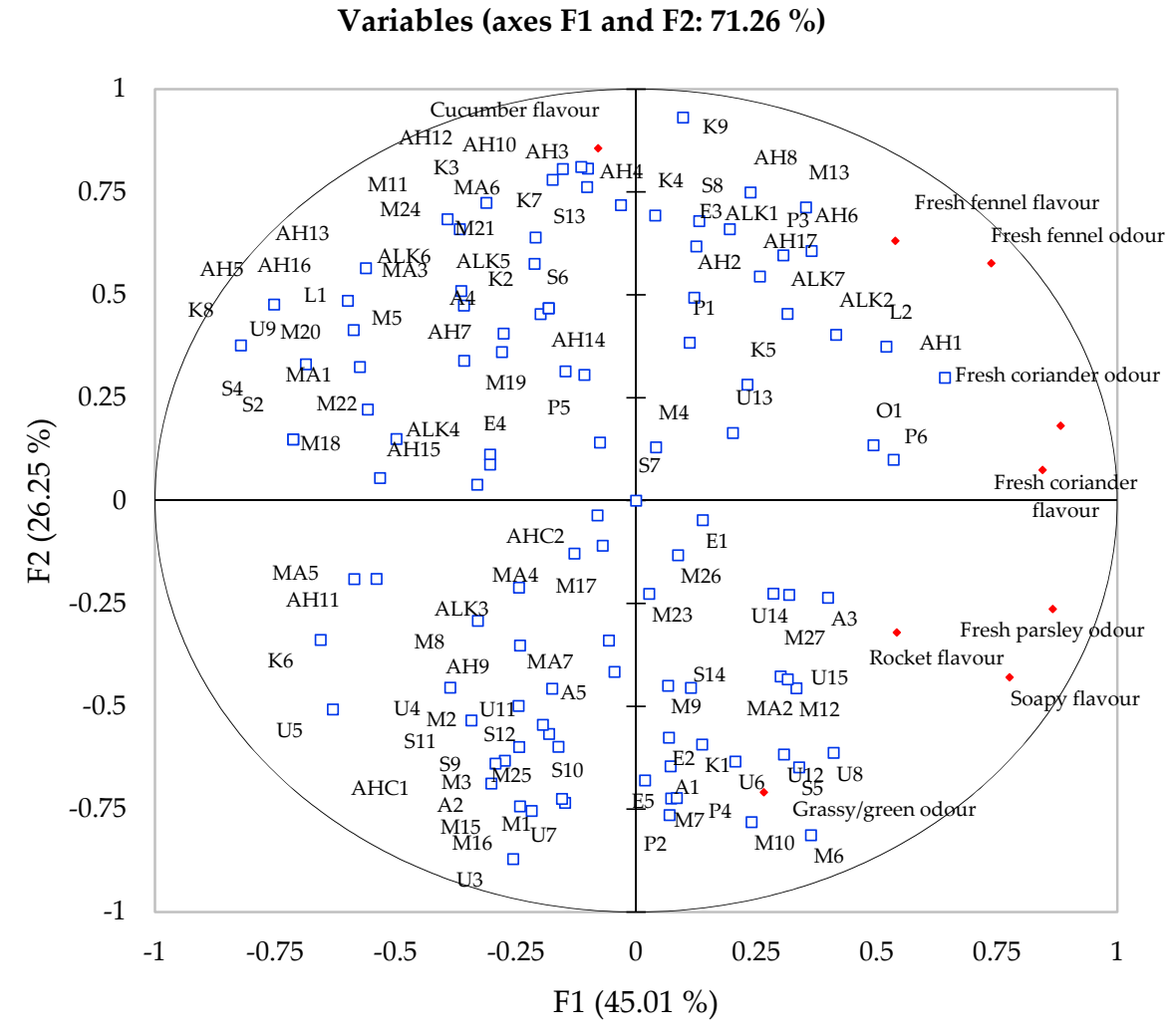

(B)

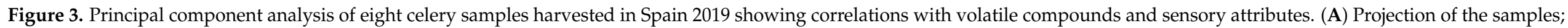
(B) Distribution of variables. 
Principal component one (F1) and two (F2) explained 71.26\% of total variation observed within the dataset for the samples grown in Spain, and the first axis separated genotypes 10, 12 and 22, whereas genotypes 5, 12, 22 and 25 are separated along the second axis. Genotype 25 in Spain exhibited a low association to all attributes apart from cucumber flavour, observed in UK 25, and genotype 12 in Spain expressed a significant association to grass odour, as observed in the UK. Furthermore, genotype 18 displayed a positive association with fresh coriander and fennel odour and flavour attributes when grown in Spain and the UK. The perception of genotypes 5, 8, 10, 15 and 22 was observed to change significantly between locations, caused by the chemical compositional changes.

The flavour attribute of cucumber displayed no significant correlations in UK compounds (Figure 2), yet significant correlations between compounds and this attribute were observed with multiple aldehydes (AH3, AH5, AH10, AH12 and AH13) that express odour characteristics such as fatty, cucumber and green (Figure 3). These compounds were not identified in the UK harvest. Compounds identified in UK celery (Figure 2) all displayed association with a flavour/odour attribute of sorts; however, this was not reflected within Spanish-grown celery. Plotto et al. [48] calculated the retronasal and orthonasal activity values for selected terpenes and aldehydes in an orange juice matrix, identifying limonene, $\beta$-pinene and $\gamma$-terpinene to have the highest thresholds in water and orange juice, whereas hexanal, octanal and nonanal, all aldehydes identified in celery (Table 1), expressed a much lower threshold. Due to the lower proportions of monoterpenes identified in Spanishgrown celery, the flavour characteristics contributed by these aldehydes (green, waxy, cucumber, honey [8]), allowed the panel to detect these more easily. This explains the differences observed in the sensory panel between the celery grown in the UK and in Spain. Furthermore, observed on the factor plot in the bottom left quadrant (Figure 3), a large group of compounds displayed no significant associations with any sensory attribute.

Celery harvested in Spain expressed a different aroma profile when compared to samples harvested in the UK, as observed in the significant difference of the aroma composition (Table 1), and although we cannot compare statistically UK and Spanish genotypes, differences in the scoring of attributes were observed. Genotypes 5, 8 and 15 displayed no association with herbal odour and flavour attributes in the UK (Figure 2) but were scored higher after growing in Spain, where strong associations to fresh fennel, coriander and parsley were displayed (Figure 3). Genotype 12 expressed close association with grass and fresh parsley odours, in addition to sedanenolide and 3-n-butylphthalide, compounds known for their celery odours, and displayed significant positive correlations with grass and parsley odour. On the other hand, genotype 25 expressed the lowest relative content of volatile compounds identified, apart from aldehyde compounds, and was scored with a significantly higher cucumber flavour than any other genotype in both locations. Here, we can assume this genotype does not exhibit a strong characteristic odour in comparison to genotype 12. As both these genotypes performed in a similar manner across locations, we would recommend these genotypes to breeders and fresh produce growers who plan to use the same cultivar across different locations, as they have expressed stability in volatile composition.

\subsection{Environmental Differences between Geographical Location and Influence on the Aroma Profile}

In this study, differences in the volatile composition and sensory profile were observed between eight genotypes and two geographical locations. Previously, Turner et al. [10] used the same genotypes grown in different years in the UK and identified that differences in temperatures (air and soil) played an important role in determining the overall flavour of celery. Environmental data including temperature, rainfall and relative humidity were collected at the nearest weather station to the farm of growth and provided by G's Fresh UK and Grupo G's España (Table 3) to compare the differences in the climate of geographical location. These environmental and geographical differences and how they influence the chemical composition of celery are only hypothesized due to the inadequate study of different growing conditions on celery. However, abiotic stresses from factors including 
temperature, humidity, water and mineral availability have been commonly observed in literature to influence secondary metabolic profiles in plants [49-51].

Table 3. Environmental data recorded at the nearest weather station to the farm of growth and provided by G's Fresh (UK) and Grupo G's España.

\begin{tabular}{|c|c|c|c|c|c|c|c|c|c|c|}
\hline \multirow[b]{2}{*}{$\begin{array}{l}\text { Weeks after } \\
\text { Transplant }\end{array}$} & \multirow[b]{2}{*}{$\begin{array}{l}\text { Air Temp } \\
\quad\left({ }^{\circ} \mathrm{C}\right)\end{array}$} & \multicolumn{3}{|c|}{ Ely, Cambridgeshire (UK) } & \multicolumn{6}{|c|}{ Aguilas, Mercia (Spain) } \\
\hline & & $\begin{array}{l}\text { Rainfall } \\
\text { (mm) }\end{array}$ & $\begin{array}{c}\text { Relative } \\
\text { Humidity } \\
(\%)\end{array}$ & $\begin{array}{l}\text { Wind } \\
\text { Speed } \\
(\mathrm{m} / \mathrm{s})\end{array}$ & $\begin{array}{l}\text { Dew } \\
\text { Point } \\
\left({ }^{\circ} \mathrm{C}\right)\end{array}$ & $\begin{array}{c}\text { Air Temp } \\
\left({ }^{\circ} \mathrm{C}\right)\end{array}$ & $\begin{array}{c}\text { Rainfall } \\
(\mathrm{mm})\end{array}$ & $\begin{array}{c}\text { Relative } \\
\text { Humidity } \\
(\%)\end{array}$ & $\begin{array}{c}\text { Wind } \\
\text { Speed } \\
(\mathrm{m} / \mathrm{s})\end{array}$ & $\begin{array}{c}\text { Dew } \\
\text { Point } \\
\left({ }^{\circ} \mathrm{C}\right)\end{array}$ \\
\hline 1 & 17.0 & 0.0 & 73.0 & 2.4 & 15.4 & 15.3 & 0.0 & 79.6 & 0.8 & 1.9 \\
\hline 2 & 14.7 & 0.0 & 81.3 & 1.5 & 18.7 & 15.4 & 0.1 & 76.3 & 1.1 & 3.9 \\
\hline 3 & 16.4 & 0.1 & 66.1 & 1.3 & 20.0 & 19.9 & 0.0 & 72.8 & 2.4 & 4.1 \\
\hline 4 & 17.0 & 0.0 & 94.8 & 1.6 & 18.4 & 17.4 & 0.1 & 63.7 & 2.9 & 1.1 \\
\hline 5 & 18.9 & 0.0 & 98.5 & 1.5 & 20.4 & 16.9 & 0.0 & 82.1 & 1.0 & 6.9 \\
\hline 6 & 19.8 & 0.0 & 99.7 & 3.0 & 16.3 & 16.4 & 0.0 & 81.2 & 1.9 & 6.1 \\
\hline 7 & 18.2 & 0.0 & 99.4 & 1.4 & 6.5 & 16.6 & 0.0 & 82.5 & 1.2 & 6.3 \\
\hline 8 & 20.4 & 0.0 & 99.0 & 1.9 & 16.3 & 18.5 & 0.0 & 84.7 & 0.8 & 8.2 \\
\hline 9 & 21.4 & 0.1 & 70.5 & 2.1 & 18.2 & 18.9 & 0.0 & 78.3 & 1.3 & 6.9 \\
\hline 10 & 20.9 & 0.0 & 71.8 & 2.6 & 13.9 & 19.8 & 0.0 & 79.4 & 1.4 & 7.2 \\
\hline 11 & 17.3 & 0.2 & 99.9 & 1.0 & 12.4 & 17.9 & 0.3 & 71.1 & 2.2 & 5.1 \\
\hline 12 & 18.4 & 0.0 & 98.6 & 2.3 & 12.9 & 16.9 & 1.8 & 78.3 & 2.1 & 8.0 \\
\hline 13 & 15.8 & 0.0 & 93.9 & 2.0 & 12.4 & 19.0 & 0.6 & 74.3 & 2.4 & 6.6 \\
\hline Average & 18.2 & 0.0 & 88.1 & 1.9 & 15.5 & 17.6 & 0.4 & 77.3 & 1.7 & 6.0 \\
\hline
\end{tabular}

Utilising two seasons for growing and using the same eight genotypes, Turner et al. [10] identified that warmer temperatures had a positive correlation with sesquiterpene and phthalide generation, whereas growing in lower temperatures led to celery with a higher monoterpene content. As similarly discussed by the authors [10], data from two harvests are insufficient when stating any relationships between environment and volatile composition, however, collating the data collected in this investigation, the dataset is completed with eight genotypes in a multi-site and multi-year experiment. Similarities in the chemical profile were observed in genotypes 12, 18, 22 and 25 in how they reacted to being grown in an alternative environment, suggesting that genotype predetermines the protective or coping mechanisms for the crop when exposed to abiotic and biotic stresses.

Celery grown in 2018 in the UK was subjected to temperatures much warmer than considered normal for the UK, and the environmental values do not express any significant differences between geographical location (Table 3) apart from the dew point; UK grown celery was grown in an environment where the average dew point value was $15.5^{\circ} \mathrm{C}$, substantially higher when compared to the $5.7^{\circ} \mathrm{C}$ experienced by Spanish-grown celery. The observed dew point temperature indicates the temperature required for the air to cool to reach a relative humidity of $100 \%$. The average daily temperature of UK grown celery is $18.2{ }^{\circ} \mathrm{C}$ and much closer to the dew point value, confirming the increased humidity experienced by UK grown celery. Exposure to high dew points promotes the growth of pathogens, inhibiting crop growth and, subsequently, compromising the crop to biotic stresses [52]. Specific stresses such as those caused by a pathogen will cause the crop to prepare a stress response and, additionally, increase the rate of plant-to-plant signalling as a form of communication, perhaps explaining the increased content of monoterpene compounds observed by the UK grown crop (Table 1). Sampaio, Edrada-Ebel and Da Costa [53] studied the influence of environmental factors on the secondary metabolic profile of Tithonia diversifolia, observing a variation within the metabolic profile in the leaves and stems, expressing a stronger association with rainfall and humidity levels than with temperature and solar radiation. The primary metabolite content of Tithonia diversifolia expressed a strong positive correlation with relative humidity, whereas secondary metabolite content expressed a strong negative correlation with humidity. A similar reaction was observed in the present study, whereby more secondary metabolites in the form of volatile compounds were identified in Spanish grown celery, where relative humidity was lower (Table 3). 
Due to minimal differences in the climate data, investigating differences in agriculture, including water and soil composition, must be included in the discussion, as these factors will also influence the flavour outcome. As a consequence of the arid and semi-arid conditions of Aguilas, Spain and the increasing shortage of water for crop irrigation, desalinated seawater is often used in southern regions of Spain [54]. Conversely, the crop irrigation system in place within the UK is by fresh water from a nearby reservoir, supplied by the river Little Ouse, in this instance. Although rigorous pre-treatment processing and filtration steps would have been completed upon both water supplies, the mineral composition of water will be vastly diverse due to differences in the original source. This will lead to variances in the soil for uptake in minerals such as calcium, sodium, magnesium, zinc and iron.

Growing in different geographical locations involves growing on different soil types. This will lead to differences in the soil properties including water holding capacity and mineral composition. UK celery was grown on loamy and sandy soils with naturally high groundwater, allowing for high water availability and nutrient uptake, whereas the Calcisol soils of Spain are known for their accumulation of calcium carbonate from precipitation brought about by evaporation under arid and semi-arid conditions [55]. The presence of surplus calcium carbonate in the soil could ultimately cause a stress response by the crop. To promote healthy growth, the crop must uptake soil, waterborne micronutrients and inorganic elements which are necessary for functional growth and involved in an array of essential pathways, including the synthesis of secondary metabolites such as isoprenoid through the non-mevalonate pathway, i.e., the building block for monoterpenes and sesquiterpenes. Primarily, carbon-, nitrogen-, sulphur- and phosphorous-fixation is involved in the synthesis of substrates and precursors involved in primary and secondary metabolism [56]. The micronutrient and element content of the soil and its permeability will influence the uptake of water and minerals from the soil to be utilised within the crop. These micronutrients can be applied by the plant for a range of uses; for example, copper has been identified to improve the flavour of fruits and vegetables along with increasing sugar and lignin content, zinc promotes the transformation and consumption of carbohydrates in plants and iron is a prominent micronutrient involved in the synthesis of organic acids [57,58]. Applying fertilisers (organic or inorganic) will increase the soil micronutrient content leading to the desired elements being available for crop uptake. Calcium and boron deficiencies, known causes of black heart and hollow stem in celery, are both nutrientdeficient illnesses that can be avoided through the application of appropriate sprays and fertiliser [59]. However, van Wassenhove, Dirinck, Schamp and Vulsteke [12] identified the negative impact of using nitrogen-based fertilizer on celery and its volatile composition. Contrary to what has been discussed above, an increased application of a nitrogen fertilizer (organic and/or mineral nitrogen) led to a reduction in the aroma-determining compounds in two celery cultivars. In fact, applying no fertilizer resulted in a higher content of volatile compounds including phthalides, whereas an overall decrease was observed between 1000 and $2000 \mu \mathrm{g} \mathrm{kg}^{-1}$ of fresh material when a nitrogen fertilizer was applied. D'Antuono, Neri and Moretti [60], similarly, observed a decrease in volatile content as nitrogen fertilizer volume was increased, especially in compounds such as limonene, myrcene and $\beta$-selinene. However, total phthalide content along with $\beta$-caryophyllene and $\alpha$-selinene were identified in high proportions when $300 \mathrm{~kg} \mathrm{ha}^{-1}$ of nitrogen was used on celery. It is possible that Spanish grown celery was exposed to higher levels of nitrogen, thus leading to a lower proportion of monoterpenes, sesquiterpenes and phthalides within the aroma composition.

Factors that accompany field placement will be a less significant cause of variation, but when these factors are combined, they will play a more significant role in determining the secondary metabolite content in celery. Possibly the most obvious difference between geographical location would be the altitude of each field: UK celery was grown on an east-facing field that was -1 to $1 \mathrm{~m}$ above sea level, whereas the field in Aguilas was south-facing at $390 \mathrm{~m}$ above sea level. Higher altitudes will result in lower temperatures 
and limitations on light exposure [61]. Cui et al. [61] investigated the physiological changes of Leymus secalinus and the effect of altitude, observing an increase in soluble sugars as elevation increased but a decrease in chlorophyll $a$ and $b$, leading to a decrease in the crop's ability to absorb light. Both these reactions were noted as defence mechanisms and adaption strategies to the change in environment. It is possible that these environmental differences led the Spanish celery to synthesise ketones and aldehydes in response to these abiotic stresses. The solar radiation would be significantly higher in the UK-grown celery due to the lower altitude along with growing in the summer months. This will increase the duration of light exposed to the crop and, thus, increase the rate of photosynthesis. Although not discussed in celery, higher exposure to UV-B in tree foliage led to an increase in flavonoids as a protective mechanism [62], and it is possible that a similar mechanism occurred in UK celery but for monoterpene production.

Synthesising aromatic compounds is a typical response from the crop to abiotic and biotic stresses for protection and adaption to the growing environment, and it is clear the celery grown in the UK reacted differently to the celery grown in Spain. Turner et al. [10] previously suggested that increased sesquiterpene and phthalide content was due to temperature stress, yet similar temperatures and other climate conditions were experienced by the Spanish crop, leading to variation in the chemical composition. Differences in soil, water and fertilizer composition used upon the UK- and Spanish-grown celery caused a change in the availability of minerals and elements to be used for primary and secondary metabolite production and, along with the placement of the field which altered the duration of light, caused a change in the crop's defence mechanism and adaption strategy.

\section{Conclusions}

Geographical location displayed a strong influence over the aroma composition of eight celery genotypes, and the influence expressed by genotype remained significant. Changes in composition caused by these factors led to differences in the aroma profile and, hence, sensory differences between genotypes and celery grown in different geographical locations were identified. Completing volatile analysis and sensory evaluation of the eight genotypes of celery demonstrated that celery genotypes grown and harvested in the UK were perceived with a strong green aroma and cucumber flavour compared to the celery grown and harvested in Spain. A wider range of compound families were identified within Spanish celery samples, imparting a significantly different aroma profile, which was perceived to be more closely associated with fresh fennel and coriander flavour. Identifying more compounds, including aldehydes and ketones in Spanish-grown celery, allowed for the explanation of the association to cucumber flavour.

Combining findings presented in this study and in the previous study completed by the authors [10], the genetic make-up of the crop regulates the synthesis of primary and secondary metabolites in response to abiotic and biotic stresses. Nonetheless, the environmental stresses experienced by the UK and Spanish crops were different and, thus, a different defence mechanism was required. This was reflected by the number of compounds expressing significant differences between genotypes and the variation caused by genotype in the UK crop, as well as the variation in perception between genotypes from sensory evaluation. The influence of geographical location on the aroma composition was also evident through the variation observed due to the location, in addition to most compounds also expressing significant differences caused by geographical location. The chemical composition was different in both locations, mostly caused by the aldehyde and ketone contents that were expressed in a significantly higher proportion of the volatile composition when sampling celery grown in Spain. A similar response was observed between harvest years, whereby significant compositional differences when the warmer temperatures of 2018 celery were observed, ultimately leading to an increased sesquiterpene and phthalide content in the eight genotypes when grown in a considerably warmer climate in response to stress. 
All eight genotypes used within these studies were observed to be influenced by both genotype and external factors, including the environment (air temperatures, soil temperatures, relative humidity), geographical location (altitude and placement of field) and agronomic techniques (application of fertilisers, water availability and irrigation systems). Two genotypes (12 and 25) demonstrated consistency in their performance across harvest year and location; 12 remained a high "extreme", profiled with strong fresh coriander and fennel attribute notes, which was reflected through its abundance in strong aroma compounds. On the other hand, genotype 25 was presented as a low "extreme" and was only profiled with a cucumber flavour, expressing significant correlations with related compounds, predominantly, aldehydes and ketones. This consistency makes these lines strong candidates to drive breeding programmes aimed at developing celery with distinct flavour profiles that will appeal to different consumer groups.

With apparent differences in the aroma and sensory profile, identifying which harvest year, environment, geographical location and agronomy produced the most appealing celery is impossible to accomplish without carrying out consumer preference trials combined with sensory profiling. Combining the data collected from this study and experiences alike with consumer preference tests would aid in the identification of attributes that consumers find important in celery products, including preferences for sweet, bitter and flavour intensity. The findings from this study could be offered to celery breeders and fresh produce growers to guide celery production with aroma profile targets in mind. Furthermore, by educating breeders about the environment, including location, genotype and agronomy, a deeper understanding will be provided on the role these factors play in determining and influencing the aroma profile and, therefore, the sensory perception of celery. Combining all these considerations will lead to a higher quality and better tasting product. Additionally, selecting cultivars according to the growing environment rather than using the same cultivar across circumstances will allow for a more consistent product.

Supplementary Materials: The following are available online at https://www.mdpi.com/article/10 $.3390 /$ ijms222112016/s1.

Author Contributions: Conceptualization, L.T., C.W., F.G. and S.L.; methodology, L.T. and S.L.; software, L.T. and S.L.; validation, L.T. and S.L.; formal analysis, L.T.; investigation, L.T.; resources, L.T.; data curation, L.T.; writing—original draft preparation, L.T.; writing—review and editing, L.T., F.G., C.W. and S.L.; supervision, S.L., F.G. and C.W.; funding acquisition: F.G. and C.W. All authors have read and agreed to the published version of the manuscript.

Funding: L.T. is funded by a BBSRC CASE PhD studentship reference BB/M016579/1 in partnership with A.L. Tozer Ltd. F.G. is employed by the company A.L. Tozer Ltd.

Institutional Review Board Statement: Ethical review and approval were not necessary for this study as the study involved tasting fresh celery samples harvested under standard commercial practices by a trained sensory panel, the members of which are employees and have consented to taste and rate food as part of their job. Ethics approval and separate consent is only required from the trained panel where they are tasting non-standard, non-commercial or novel food ingredients. The trained panel work was within the ethical and professional practices set out by the IFST: https: / lwww.ifst.org/membership/networksand-communities/special-interest-groups / sensory-science-group/ifst-guidelines (accessed on 4 November 2021).

Informed Consent Statement: Our trained employed sensory panel provided consent to taste and rate food as part of their job; they only provide separate written consent to a specific study where they are tasting non-standard, non-commercial or novel food ingredients.

Data Availability Statement: The data presented in this study are available upon request from the corresponding author. 
Acknowledgments: L.T. would like to thank G's Fresh Ltd. for growing the celery crops and Sara Jennings and Isobel Tickner for their help with overseeing the trials and the celery harvests.

Conflicts of Interest: The funders discussed the design of the study with the research team, but they were not involved in the collection or analysis of data. Frances Gawthrop is a co-author on this paper; she reviewed the manuscript and agreed to publish the results. The other authors declare no conflict of interest.

\section{References}

1. Rożek, E. Growth and yielding of leaf celery (Apium graveolens L.var. secalinum Alef.) cultivated for two-cut harvest. Herba Polonica 2007, 53, 17-21.

2. Malhotra, S. Celery. In Handbook of Herbs and Spices, 2nd ed.; Peter, K., Ed.; Woodhead Publishing Ltd.: Cambridgshire, UK, 2012; Volume 2, pp. 249-267.

3. Uhlig, J.W.; Chang, A.; Jen, J.J. Effect of Phthalides on Celery Flavor. J. Food Sci. 1987, 52, 658-660. [CrossRef]

4. Jegorova, A.; Kailas, T.; Orav, A. Composition of the essential oil of dill, celery, and parsley from Estonia. Proc.-Est. Acad. Sci. Chem. 2003, 52, 147. [CrossRef]

5. Sellami, I.; Bettaieb, I.; Bourgou, S.; Dahmani, R.; Limam, F.; Marzouk, B. Essential oil and aroma composition of leaves, stalks and roots of celery (Apium graveolens var. dulce) from Tunisia. J. Essent. Oil Res. 2012, 24, 513-521. [CrossRef]

6. MacLeod, G.; Ames, J.M. Volatile components of celery and celeriac. Phytochemistry 1989, 28, 1817-1824. [CrossRef]

7. Turner, L.; Lignou, S.; Gawthrop, F.; Wagstaff, C. Investigating the factors that influence the aroma profile of Apium graveolens: A review. Food Chem. 2020, 345, 128673. [CrossRef]

8. Turner, L.; Dawda, D.; Wagstaff, C.; Gawthrop, F.; Lignou, S. Influence of harvest maturity on the aroma quality of two celery (Apium graveolens) genotypes. Food Chem. 2021, 365, 130515. [CrossRef]

9. Gold, H.J.; Wilson, C.W. The Volatile Flavor Substances of Celery. J. Food Sci. 1963, 28, 484-488. [CrossRef]

10. Turner, L.; Lignou, S.; Gawthrop, F.; Wagstaff, C. Investigating the Relationship of Genotype and Climate Conditions on the Volatile Composition and Sensory Profile of Celery (Apium graveolens). Foods 2021, 10, 1335. [CrossRef]

11. Marongiu, B.; Piras, A.; Porcedda, S.; Falconieri, D.; Maxia, A.; Frau, M.; Gonçalves, M.; Cavaleiro, C.; Salgueiro, L. Isolation of the volatile fraction from Apium graveolens L. (Apiaceae) by supercritical carbon dioxide extraction and hydrodistillation: Chemical composition and antifungal activity. Nat. Prod. Res. 2012, 27, 1521-1527. [CrossRef]

12. Rożek, E.; Nurzyńska-Wierdak, R.; Kosior, M. Efficiency of some agrotechnical treatments in quantity and quality yield modification of leaf celery (Apium graveolens L. var. Secalinum Alef). Acta Sci. Pol. Hortorum Cultus 2013, 12, $227-239$.

13. Van Wassenhove, F.A.; Dirinck, P.J.; Schamp, N.M.; Vulsteke, G.A. Effect of nitrogen fertilizers on celery volatiles. J. Agric. Food Chem. 1990, 38, 220-226. [CrossRef]

14. Lisiewska, Z.; Kmiecik, W. Dependence of dried chive (Allium schoenoprasum) quality upon the drying method and storage period. EJPAU 1998, 1, 6 .

15. Díaz-Maroto, M.C.; Palomo, E.S.; Castro, L.; Viñas, M.G.; Pérez-Coello, M.S. Changes produced in the aroma compounds and structural integrity of basil (Ocimum basilicum L.) during drying. J. Sci. Food Agric. 2004, 84, 2070-2076. [CrossRef]

16. Rołson, W.; Osińska, E.; Wajs-Bonikowska, A. Effect of plantation establishment and raw material stabilization on the usefull traits of lovage leaves (Levisticum officinale Koch.). Acta Sci. Pol. Hortorum Cultus 2013, 12, 141-155.

17. Hoffmann, M. Sensory quality of selected freeze-dried and dried seasoning vegetables in food. Nauka Technol. Jakość 2007, 2, 91-97.

18. Radulović, N.; Blagojević, P.; Palić, R. Comparative Study of the Leaf Volatiles of Arctostaphylos uva-ursi (L.) Spreng. and Vaccinium vitis-idaea L. (Ericaceae). Molecules 2010, 15, 6168-6185. [CrossRef]

19. Andriamaharavo, N.R. Retention Data. NIST Mass Spectrometry Data Center. 2014. Available online: https://webbook.nist.gov/ cgi / cbook.cgi? Source=2014AND\%2319410M\&Units=SI\&Mask=2000 (accessed on 8 July 2021).

20. Stashenko, E.; Jaramillo, B.; Martínez, J. Comparación de la composición química y de la actividad antioxidante in vitro de los metabolitos secundarios volátiles de plantas de la familia verbenaceae. Rev. Acad. Colomb. Cienc. Exactas Fis. Nat. $2003,27,579-597$.

21. Lucero, M.; Fredrickson, E.; Estell, R.; Morrison, A.; Richman, D. Volatile Composition of Gutierrezia sarothrae (Broom Snake-weed) as Determined by Steam Distillation and Solid Phase Microextraction. J. Essent. Oil Res. 2006, 18, 121-125. [CrossRef]

22. Beaulieu, J.C.; Grimm, C.C. Identification of Volatile Compounds in Cantaloupe at Various Developmental Stages Using Solid Phase Microextraction. J. Agric. Food Chem. 2001, 49, 1345-1352. [CrossRef]

23. Lucero, M.E.; Estell, R.E.; Frederickson, E.L. The essential oil composition of Psorothamnus scoparius (A. Gray) Rydb. J. Essent. Oil Res. 2003, 15, 108-111. [CrossRef]

24. Adams, R.P.; Morris, J.A.; Pandey, R.N.; Schwarzbach, A.E. Cryptic speciation between Juniperus deltoides and Juniperus oxycedrus (Cupressaceae) in the Mediterranean. Biochem. Syst. Ecol. 2005, 33, 771-787. [CrossRef]

25. Sabulal, B.; Dan, M.; John, A.; Kurup, R.; Chandrika, S.; George, V. Phenylbutanoid-rich rhizome oil of Zingiber neesanum from Western Ghats, southern India. Flavour Fragr. J. 2007, 22, 521-524. [CrossRef]

26. Havlik, J.; Kokoska, L.; Vasickova, S.; Valterova, I. Chemical composition of essential oil from the seeds of Nigella arvensis L. and assessment of its antimicrobial activity. Flavour Fragr. J. 2006, 21, 713-717. [CrossRef]

27. Bylaite, E.; Meyer, A.S. Characterisation of volatile aroma compounds of orange juices by three dynamic and static headspace gas chromatography techniques. Eur. Food Res. Technol. 2005, 222, 176-184. [CrossRef] 
28. Block, S.; Flamini, G.; Brkic, D.; Morelli, I.; Quetin-Leclercq, J. Analysis of the essential oil from leaves of Croton zambesicus Muell. Arg. growing in Benin. Flavour Fragr. J. 2006, 21, 222-224. [CrossRef]

29. Boulanger, R.; Chassagne, D.; Crouzet, J. Free and bound flavour components of amazonian fruits. 1: Bacuri. Flavour Fragr. J. 1999, 14, 303-311. [CrossRef]

30. Cao, H.; Li, Z.; Chen, X. QSRR Study of GC Retention Indices of Volatile Compounds Emitted from Mosla chinensis Maxim by Multiple Linear Regression. Chin. J. Chem. 2011, 29, 2187-2196. [CrossRef]

31. Yu, Y.; Huang, T.; Yang, B.; Liu, X.; Duan, G. Development of gas chromatography-mass spectrometry with microwave dis-tillation and simultaneous solid-phase microextraction for rapid determination of volatile constituents in ginger. J. Pharm. Biomed. Anal. 2007, 43, 24-31. [CrossRef]

32. Zeng, Y.-X.; Zhao, C.-X.; Liang, Y.-Z.; Yang, H.; Fang, H.-Z.; Yi, L.-Z.; Zeng, Z.-D. Comparative analysis of volatile components from Clematis species growing in China. Anal. Chim. Acta 2007, 595, 328-339. [CrossRef]

33. Javidnia, K.; Miri, R.; Kamalinejad, M.; Mehdipour, A.R. Composition of the essential oil of Diplotaenia cachrydifolia Boiss from Iran. J. Ess. Oil Res. 2006, 18, 86-87. [CrossRef]

34. Pripdeevech, P.; Saansoomchai, J. Antibacterial activity and chemical composition of essential oil and various extracts of Fagraea fragrans Roxb. flowers. Chiang Mai J. Sci. 2013, 40, 214-223.

35. Ansorena, D.; Gimeno, O.; Astiasarán, I.; Bello, J. Analysis of volatile compounds by GC-MS of a dry fermented sausage: Chorizo de Pamplona. Food Res. Int. 2001, 34, 67-75. [CrossRef]

36. Philippe, J.; Suvarnalatha, G.; Sankar, R.; Suresh, S. Kessane in the Indian Celery Seed Oils. J. Essent. Oil Res. 2002, 14, 276-277. [CrossRef]

37. Van Wassenhove, F.; Dirinck, P.; Vulsteke, G.; Schamp, N. Aromatic Volatile Composition of Celery and Celeriac Cultivars. HortScience 1990, 25, 556-559. [CrossRef]

38. Kurobayashi, Y.; Kouno, E.; Fujita, A.; Morimitsu, Y.; Kubota, K. Potent Odorants Characterize the Aroma Quality of Leaves and Stalks in Raw and Boiled Celery. Biosci. Biotechnol. Biochem. 2006, 70, 958-965. [CrossRef]

39. Pino, J.; Rosado, A.; Fuentes, V. Leaf oil of Celery (Apium graveolens L.) from Cuba. J. Essent. Oil. Res. 1997, 9, 719-720. [CrossRef]

40. Shojaei, Z.A.; Ebrahimi, A.; Salimi, M. Chemical Composition of Three Ecotypes of Wild Celery (Kelussia odoratissima). J. Herbs Spices Med. Plants 2011, 17, 62-68. [CrossRef]

41. Lund, E.; Wagner, C.; Bryan, W. Oils recovered from celery packinghouse waste. Florida State Hortic. Soc. 1973, 86, $255-259$.

42. Phan, D.H.T.; Kim, B.; Dong, V.M. Phthalides by Rhodium-Catalyzed Ketone Hydroacylation. J. Am. Chem. Soc. 2009, 131, 15608-15609. [CrossRef]

43. Moya, C.; Oyanedel, E.; Verdugo, G.; Fernanda, F.M.; Urrestarazu, M.; Alvaro, J.E. Increased electrical conductivity in nu-trient solution management enhances dietary and organoleptic qualities in soilless culture tomato. HortScience 2017, 52, 868-872. [CrossRef]

44. Marin, A.; Rubio, J.S.; Martinez, V.; Gil, M.I. Antioxidant compounds in green and red peppers as affected by irrigation fre-quency, salinity and nutrient solution composition. J. Sci. Food Agric. 2009, 89, 1352-1359. [CrossRef]

45. Giuffrida, F.; Cassaniti, C.; Malvuccio, A.; Leonardi, C. Effects of salt stress imposed during two growth phases on cauliflower production and quality. J. Sci. Food Agric. 2016, 97, 1552-1560. [CrossRef] [PubMed]

46. Lignou, S.; Parker, J.K.; Baxter, C.; Mottram, D.S. Sensory and instrumental analysis of medium and long shelf-life Charentais cantaloupe melons (Cucumis melo L.) harvested at different maturities. Food Chem. 2013, 148, 218-229. [CrossRef] [PubMed]

47. Eriksson, N.; Wu, S.; Do, C.B.; Kiefer, A.K.; Tung, J.Y.; Mountain, J.L.; Hinds, D.A.; Francke, U. A genetic variant near olfactory receptor genes influences cilantro preference. Flavour 2012, 1, 22. [CrossRef]

48. Plotto, A.; Margaría, C.A.; Goodner, K.L.; Goodrich, R.; Baldwin, E.A. Odour and flavour thresholds for key aroma components in an orange juice matrix: Terpenes and aldehydes. Flavour Fragr. J. 2004, 19, 491-498. [CrossRef]

49. Ramakrishna, A.; Ravishankar, G. Influence of abiotic stress signals on secondary metabolites in plants. Plant. Signal. Behav. 2011, 6, 1720-1731.

50. Miller, G.; Shulaev, V.; Mittler, R. Reactive oxygen signaling and abiotic stress. Physiol. Plant. 2008, 133, 481-489. [CrossRef]

51. Arbona, V.; Manzi, M.; de Ollas, C.; Gómez-Cadenas, A. Metabolomics as a tool to investigate abiotic stress tolerance in plants. Int. J. Mol. Sci. 2013, 14, 4885-4911. [CrossRef]

52. Park, D.-H.; Park, J.-W. Wireless sensory network-based greenhouse environment monitoring and automatic control system for dew condensation prevention. Sensors 2011, 11,3640-3651. [CrossRef]

53. Sampaio, B.; Edrada-Ebel, R.; Da Costa, F. Effect of the environment on the secondary metabolic profile of Tithonia diversifola: A model for environmental metabolomics of plants. Sci. Rep. 2016, 6, 29265. [CrossRef]

54. Martinez-Alvarez, V.; Maestre-Valero, J.; González-Ortega, M.; Gallego, B.; Martin-Gorriz, B. Characterization of the agri-cultural supply of desalinated seawater in Southeastern Spain. Water 2019, 11, 1233. [CrossRef]

55. FAO. Lecture notes on the major soils of the world. In World Soil Resources Reports, 94; Food and Agriculture Organization of the United Nations: Rome, Italy, 2001; p. 334.

56. Waterman, P.G.; Mole, S. Extrinsic Factors Influencing Production of Secondary Metabolites in Plants. Insect Plant Ineractions 2019, 107-134. [CrossRef]

57. Mousavi, S.; Galvai, M.; Razaei, M. The interaction of zinc with other elements in plants: A review. The interaction of zinc with other elements in plants: A review. Int. J. Agric. Crop Sci. 2021, 4, 1881-1884.

58. Broadley, M.; Brown, P.; Cakmak, I.; Rengel, Z.; Zhao, F. Function of Nutrients: Micronutrients. In Marschner's Mineral Nutrition of Higher Plants, 3rd ed.; Academic Press: Cambridge, MA, USA, 2021; pp. 191-248. 
59. Rubatzky, V.E.; Quiros, C.F.; Simon, P.W. Carrots and Related Vegetable Umbelliferae; CABI Publishing: Wallingford, UK, 1999.

60. D'Antuono, L.; Neri, R.; Moretti, A. By-products of vegetable celery (Apium graveolens L. var. dulce) as potential source of flavours. Acta Hortic. 2002, 327-331. [CrossRef]

61. Cui, G.; Li, B.; He, W.; Yin, X.; Liu, S.; Lian, L.; Zhang, Y.; Liang, W.; Zhang, P. Physiological analysis of the effect of altitudinal gradients on Leymus secalinus on the Qinghai-Tibetan Plateau. PLoS ONE 2018, 13, e0202881. [CrossRef]

62. Nissinen, K.; Virjamo, V.; Randriamanana, T.; Sobuj, N.; Sivadasan, U.; Mehtätalo, L.; Beuker, E.; Julkunen-Tiitto, R.; Nybakken, L. Responses of growth and leaf phenolics in European aspen (Populus tremula) to climate change during juvenile phase change. Can. J. For. Res. 2017, 47, 1350-1363. [CrossRef] 